\title{
1 Glycomic profiling of the gut microbiota by Glycan-seq
}

2 Lalhaba Oinam ${ }^{1}$, Fumi Minoshima ${ }^{1}$, and Hiroaki Tateno ${ }^{1,2^{*}}$

$3{ }^{1}$ Cellular and Molecular Biotechnology Research Institute, National Institute of Advanced Industrial Science

4 and Technology (AIST), Tsukuba Central 6, 1-1-1 Higashi, Tsukuba, Ibaraki 305-8566, Japan

5 22AMED-Prime, AMED, Tsukuba Central 6, 1-1-1 Higashi, Tsukuba, Ibaraki 305-8566, Japan

6

$7 \quad *$ Corresponding author:

8 Hiroaki Tateno

9 1-1-1 Higashi, Tsukuba, Ibaraki 305-8566, Japan

10 Phone and fax: $+81-29-861-3273$

11 E-mail address: h-tateno@aist.go.jp

12

13

14

15

16

17 


\section{Abstract}

19 Background: There has been immense interest in studying the relationship between the gut

20 microbiota and human health. Bacterial glycans modulate the cross talk between the gut

21 microbiota and its host. However, little is known about these glycans because of the lack of

22 appropriate technology to study them.

23 Methods: We previously developed a sequencing-based glycan profiling method called Glycan-

24 seq, which is based on the use of 39 DNA-barcoded lectins. In this study, we applied this

25 technology to analyze the glycome of the intact gut microbiota of mice. Fecal microbiota was

26 incubated with 39 DNA-barcoded lectins exposed to UV, and the number of released DNA

27 barcodes were counted by next-generation sequencing to obtain a signal for each lectin bound to

28 the microbiota. In parallel, the bacterial composition of the gut microbiota was analyzed by $16 \mathrm{~S}$

29 rRNA gene sequencing. Finally, we performed a lectin pull-down experiment followed by $16 \mathrm{~S}$

30 rRNA gene sequencing to identify lectin-reactive bacteria.

31 Results: The evaluation of cultured gram-positive (Deinococcus radiodurans) and gram-negative

32 (Escherichia coli) bacteria showed significantly distinct glycan profiles between these bacteria,

33 which were selected and further analyzed by flow cytometry. The results of flow cytometry

34 agreed well with those obtained by Glycan-seq, indicating that Glycan-seq can be used for 
35 bacterial glycomic analysis. We thus applied Glycan-seq to comparatively analyze the glycomes

36 of young and old mice gut microbiotas. The glycomes of the young and old microbiotas had

37 significantly distinct glycan profiles, which reflect the different bacterial compositions of young

38 and old gut microbiotas based on 16S rRNA gene sequencing. Therefore, the difference in the

39 glycomic profiles between young and old microbiotas may be due to their differing bacterial

40 compositions. a2-6Sia-binders bound specifically to the young microbiota. Lectin pull-down

41 followed by $16 \mathrm{~S}$ rRNA gene sequencing of the young microbiota identified Lactobacillaceae as

42 the most abundant bacterial family with glycans reacting with $\alpha 2-6$ Sia-binders.

43 Conclusion: The Glycan-seq system can, without any prior culturing and fluorescence labeling,

44 reveal the glycomic profile of the intact bacterial gut microbiota. A combination of lectin pull-

45 down and 16S rRNA gene sequencing can identify lectin-reactive bacteria.

46 Keywords: microbiota, glycome, aging, glycans, Glycan-seq, 16S rRNA sequencing, sialylation,

47 Sia 


\section{Background}

53 The microbiota of the digestive tract [1] is dominated by bacteria. It is estimated that 1000

54 species of commensal, symbiotic, and pathogenic bacteria are present in the gut microbiota [2,

55 3]. The gut microbiota plays vital roles in human health and disease conditions and is tightly

56 regulated by the lifestyle, dietary habits, and health status of the host [4]. It interacts with the gut

57 epithelium, including the different immune cells within it [5]. The gut microbiota may play

58 regulatory roles in mood, anxiety, and cognition via the gut-brain axis [6], and an imbalance in

59 the gut microbiota may cause gastrointestinal disorders [4, 7] and metabolic [8] and

60 inflammatory diseases [9].

61 The surface of the bacteria is coated with an intricate network of glycans that act as an interface

62 between mammalian hosts and their gut bacteria [10]. Gram-positive bacterial cells are enclosed

63 by a single membrane covered by a thick peptidoglycan layer and lipoteichoic acids [11],

64 whereas gram-negative bacteria are covered by two cell membranes (inner and outer membranes)

65 separated by a periplasm containing a thin peptidoglycan layer and $\beta$-glucan; the outer

66 membrane consists of lipopolysaccharides [12]. Both types of bacteria are often further enclosed

67 by a diverse array of capsular polysaccharides [13]. We previously developed a method to

68 analyze the bacterial cell surface glycomes using a lectin microarray and applied this method to

69 compare 16 different strains of Lactobacillus casei [14]. Interestingly, cell surface glycomes 
70 differ depending on the bacterial strains. However, there are several drawbacks in the lectin

71 microarray analysis of bacteria, including the following: (1) A large number of cells $\left(0.5 \times 10^{9}-5\right.$

$72 \times 10^{9}$ cells/well) are required for the analysis. (2) Bacteria bound to the lectin microarray are

73 easily released by washing steps meant to remove unbound bacteria. Thus, the results of this

74 analysis may be difficult to reproduce. (3) The analysis requires fluorescently labeled bacterial

75 cells, and different species of bacteria may differ in their fluorescence. As the gut microbiota

76 consists of various bacterial populations, labeling all bacterial populations at the same level of

77 fluorescence is difficult. Hence, the lectin microarray has never been applied to the analysis of

78 the gut microbiota. Thus, despite playing an essential role in bacterial cross talk with the host,

79 bacterial glycans in the gut microbiota remain poorly understood mainly because of insufficient

80 analysis method.

81 We recently developed a highly multiplexed glycan profiling method called Glycan-seq, which

82 analyzes bulk and single cells using DNA-barcoded lectins and next-generation sequencing [15].

83 In this study, we applied Glycan-seq to analyze the gut microbiota of mice without performing

84 any prior bacterial culturing and fluorescence labeling. First, we evaluated the applicability of

85 Glycan-seq for bacterial profiling using the cultured representatives of gram-positive and gram-

86 negative bacteria. We then used Glycan-seq to analyze the glycome alteration on the gut

87 microbiota of young and old mice. Further, 16S rRNA gene sequencing was performed to 
88 analyze the differences in the bacterial composition of the gut microbiotas in young and old

89 mice.

\section{Methods}

\section{Microbial culture}

92 Escherichia coli (Migula) (ATCC 700926) was cultured overnight at $37^{\circ} \mathrm{C}$ in $\mathrm{M} 9$ culture

93 medium, whereas Deinococcus radiodurans (ATCC BAA-816) was also cultured overnight at

$9430^{\circ} \mathrm{C}$ in TGY medium. The abundance and size of cells were analyzed using a particle counter

95 (CDA 1000; Sysmex Corporation, Hyogo, Japan).

96 Mice

97 Young (14-20 days old) and old (12 months old) C57BL/6J mice were used in this study. The

98 mice were derived or purchased from Charles River Laboratories and Japan SLC (Shizuoka,

99 Japan). Male mice were used for all the experiments. The mice were housed under specific

100 pathogen-free conditions in the Laboratory Animal Resource Center at the University of

101 Tsukuba, Japan.

102 Fecal sample collection and microbiota isolation

103 Mice were placed inside an autoclaved cage for 30-60 min, and the excreted feces were collected 
104 using sterilized forceps. The collected feces were frozen at $-20^{\circ} \mathrm{C}$ until use. The mouse fecal

105 microbiota was isolated using the density gradient method [16]. Briefly, approximately $20 \mathrm{mg}$ of

106 feces was homogenized in $0.5 \mathrm{ml}$ phosphate-buffered saline (PBS) at $4^{\circ} \mathrm{C}$ by shaking at $750 \mathrm{rpm}$

107 overnight. After homogenization, the supernatant was collected and transferred to the top of a

108 Nycodenz solution (80\% w/v in water; Cosmo Bio Co., Ltd., Tokyo, Japan). The solution was

109 then centrifuged at $10,000 \times g$ for $40 \mathrm{~min}$ at $4^{\circ} \mathrm{C}$. The middle layer containing the microbiota was

110 collected and further washed with PBS. The numbers and sizes of bacterial cells from each

111 sample were quantified using a particle counter (CDA-1000; Sysmex Corporation).

\section{Preparation of DNA-barcoded lectins}

113 Lectins were conjugated to the DNA oligonucleotide as previously described [15]. Briefly, 100

$114 \mu \mathrm{g}$ of each lectin was dissolved in $100 \mu \mathrm{l}$ of PBS mixed with dibenzocyclooctyne-N-

115 hydroxysuccinimidyl ester (DBCO-NHS) (Funakoshi Co., Ltd., Tokyo, Japan) at 10 times the

116 molar amount and then incubated in the dark for 1 hour at $20^{\circ} \mathrm{C}$. DBCO-NHS was inactivated by

117 adding $10 \mu \mathrm{l}$ of $1 \mathrm{M}$ Tris and incubating the mixture in the dark for $15 \mathrm{~min}$ at $20^{\circ} \mathrm{C}$. The excess

118 DBCO-NHS was removed using Sephadex G-25 desalting columns (GE Healthcare Japan Co.,

119 Tokyo, Japan). The DBCO-labeled lectin product (100 $\mu \mathrm{g} / \mathrm{mL})$ was mixed with 5'-azide-

120 modified DNA oligonucleotides (Integrated DNA Technologies, KK, Tokyo, Japan) at 10 times 
121 the molar amount. The conjugated lectin-DNA oligonucleotide was purified by removing

122 unbound nucleotides and selecting only the lectins with the glycan-binding affinity, which was

123 achieved by affinity chromatography using the appropriate sugar-immobilized Sepharose 4B-CL

124 (GE) based on the glycan-binding specificity of each lectin.

\section{Glycan-seq}

126 Bacterial cells $\left(1 \times 10^{7}\right)$ were suspended in PBS containing $1 \%$ bovine serum albumin $(\mathrm{BSA})$ and

127 incubated with $39 \mathrm{DNA}$-barcoded lectins at a final concentration of $0.5 \mu \mathrm{gml}^{-1}$ at $4^{\circ} \mathrm{C}$ for $1 \mathrm{~h}$.

128 The cells were washed three times with $1 \mathrm{ml}$ of PBS/BSA to liberate oligonucleotides after

129 which it was diluted ten times $\left(1 \times 10^{6}\right)$ and then were UV-irradiated at $365 \mathrm{~nm}, 15 \mathrm{~W}$, for 15

130 min using a UVP Blak-Ray XX-15L UV Bench Lamp (Analytik Jena, Kanagawa, Japan). The

131 liberated oligonucleotides were then amplified using NEBNext Ultra II Q5 (New England

132 BioLabs Japan Inc., Tokyo, Japan), i5-index, and i7-index primers containing cell

133 oligonucleotide sequences. PCR reactions were performed as follows: 1 cycle of denaturation for

$13445 \mathrm{sec}$ at $98^{\circ} \mathrm{C} ; 20$ cycles of denaturation for $10 \mathrm{sec}$ at $98^{\circ} \mathrm{C}$, followed by $50 \mathrm{sec}$ at $65^{\circ} \mathrm{C}$; and 1

135 cycle of extension for $5 \mathrm{~min}$ at $65^{\circ} \mathrm{C}$. The PCR products were then purified using the Agencourt

136 AMPure XP Kit (Beckman Coulter, Inc., Tokyo, Japan) following the manufacturer's protocol.

137 The size and quantity of the PCR products were analyzed using MultiNA (Shimadzu Co., Kyoto, 
138 Japan). The PCR products (4 nM from every sample) were treated with the MiSeq Reagent Kit

139 v2 (50 cycle format; Illumina KK, Tokyo, Japan) and sequenced using the MiSeq Sequencer (26

140 bp, paired-end) (Illumina KK, Tokyo, Japan).

141 Analysis of Glycan-seq data

142 The DNA barcodes derived from lectins were directly extracted from the reads in FASTQ format.

143 The number of DNA barcodes bound to each cell was counted using a barcode DNA counting

144 system (Mizuho Information \& Research Institute, Inc., Tokyo, Japan) [15]. The first three bases

145 in each read were removed to better match the DNA barcode sequence. In cases of mismatch, we

146 allowed a maximum of two mismatches in the flanking region and one mismatch in the middle

147 region. The total number of each of the DNA barcodes was divided by the total number of lectin

148 barcodes and expressed as a percentage (\%) for each lectin. Statistically significant levels of

149 lectins in the Glycan-seq were evaluated by $t$-tests, setting the levels of significance at $P<0.01$

150 for cultured bacteria and $P<0.05$ for the young and old gut microbiota.

\section{$151 \quad$ Flow cytometry analysis}

152 Approximately $1 \times 10^{7}$ cells of $E$. coli and D. radiodurans were incubated with $10 \mu \mathrm{g}$ of R-

153 phycoerythrin-conjugated lectins for 1 hour on ice. BSA-conjugated lectin was used as a

154 negative control. Flow cytometry data were acquired on a CytoFLEX System (Beckman Coulter, 
155 Inc., Brea, CA) and analyzed using the FlowJo software v10.6 (BD, Franklin Lakes, NJ).

156 Microbial DNA extraction from mouse feces

157 Genomic DNA was isolated from the microbial fraction collected from mouse feces (as

158 described above) by a bead-beating method implemented using the ISOSPIN Fecal DNA Kit

159 (Nippon Gene Co., Ltd, Japan). The isolated DNA was eluted in $50 \mu$ TE buffer (pH 8.0)

160 provided in the kit.

$161 \quad$ 16S rRNA gene sequencing

162 Sequencing libraries were prepared from the V3-V4 hypervariable region of 16S rRNA gene,

163 following the protocol entitled "16S Metagenomic Sequencing Library Preparation” from

164 Illumina [17]. The V3-V4 hypervariable region of 16S rRNA gene was amplified using the

165 following primers: forward: $5^{\prime}-$

166 TCGTCGGCAGCGTCAGATGTGTATAAGAGACAGCCTACGGGNGGCWGCAG-3’;

167 reverse: $5^{\prime}-$

168 GTCTCGTGGGCTCGGAGATGTGTATAAGAGACAGGACTACHVGGGTATCTAATCC-

$1693^{\prime}$ '). The $25 \mu 1$ PCR reaction was performed using a KAPA HiFi HotStart ReadyMix (Roche) and

170 contained $1 \mu \mathrm{l}$ of extracted fecal microbial DNA and $1 \mu \mathrm{M}$ of each primer. The reaction cycles

171 consisted of initial denaturation at $98^{\circ} \mathrm{C}$ for $2 \mathrm{~min}$; followed by 25 cycles of denaturation at $98^{\circ} \mathrm{C}$ 
172 for $15 \mathrm{sec}$, annealing at $56^{\circ} \mathrm{C}$ for $30 \mathrm{sec}$, and elongation at $72^{\circ} \mathrm{C}$ for $30 \mathrm{sec}$; and a final elongation

173 at $72^{\circ} \mathrm{C}$ for $5 \mathrm{~min}$.

174 Next, a second PCR was performed using Illumina index primers and the following reaction

175 cycle: initial denaturation at $95^{\circ} \mathrm{C}$ for $3 \mathrm{~min}$; followed by 8 cycles of denaturation at $95^{\circ} \mathrm{C}$ for

$17630 \mathrm{sec}$, annealing at $55^{\circ} \mathrm{C}$ for $30 \mathrm{sec}$ and elongation at $72^{\circ} \mathrm{C}$ for $30 \mathrm{sec}$; and a final elongation at

$17772^{\circ} \mathrm{C}$ for $5 \mathrm{~min}$. The amplicons were quantified using MultiNA (Shimadzu, Japan), a microchip

178 electrophoresis system for DNA/RNA analysis. The amplicons were sequenced using the

179 Illumina MiSeq $2 \times 250$ bp platform with a MiSeq Reagent Nano Kit V2 (Illumina).

\section{$180 \quad$ 16S rRNA gene sequence analysis}

181 The raw sequence reads were analyzed using QIIME2 (2020.8) [18]. The reads were first

182 demultiplexed; then, the DADA2 [19] plugin was used for quality control, read trimming, and

183 assembly. Trimming took into consideration the information needed to merge the paired reads.

184 Amplicon sequence variants (ASVs) were generated by DADA2 analysis, which were then

185 classified to family and genus levels using the q2-feature-classifier [20], a Naïve Bayes machine

186 learning classifier plugin in the QIIME2. Operational taxonomic units (OTUs) were generated by

187 the RESCRIPt QIIME2 plugin running a feature classifier trained on the V3-V4 region of the

188 16S rRNA gene using a preformatted SILVA 138 reference database [21, 22]. An equal sampling 
189 depth of 10,000 was selected for every sample for assessing the diversities. $\alpha$-diversity was

190 measured by Faith's phylogenetic diversity (PD) metrics, and significance $(p<0.05)$ was

191 statistically calculated using Kruskal-Wallis (pairwise) analysis. Using principal coordinate

192 analysis (PCoA) from the UniFrac metrics analysis, $\beta$-diversity was calculated [23].

\section{Microbe isolation by lectin}

194 SSA and TJAI lectins were labeled with biotin and used at a concentration of $1 \mu \mathrm{g} / \mathrm{ul}$. The

195 labeled lectins were incubated with streptavidin-conjugated Dynabeads (Thermo Fisher,

196 Waltham, Massachusetts, USA) in a shaker set at $1,400 \mathrm{rpm}$ at $4^{\circ} \mathrm{C}$ for 1 hour. The conjugated

197 beads were washed; then, $2 \times 10^{7}$ microbial cells from young and old mice samples were

198 incubated with the beads in a shaker at $700 \mathrm{rpm}$ at $4^{\circ} \mathrm{C}$ overnight. The bound microbes were

199 isolated using a magnetic stand and eluted with 2M lactose.

\section{Correlation analysis}

201 The $\mathrm{x}$ of lectins and the $\mathrm{y}$ of microbial communities were plotted using the corrplot package in R.

202 The analysis yielded Spearman correlation coefficients evaluated at $p<0.05$.

\section{Results}




\section{Glycomic profiling of the gut microbiota by Glycan-seq}

206 We aimed to develop a strategy to profile the glycome of the intact gut microbiota without prior

207 fluorescence labeling using Glycan-seq [15]. Cultured bacterial cells were incubated with DNA-

208 barcoded lectins, which, upon binding, released their DNA barcodes after UV exposure, because

209 lectins were conjugated with DNA barcodes via a photocleavable linker (Fig. 1). The lectins used

210 in this study cover a wide range of glycan structures, including sialylated, galactosylated,

211 GlcNAcylated, mannosylated, and fucosylated glycans (Table S1). The released DNA barcodes

212 were recovered, amplified, and analyzed by next-generation sequencing. The number of each

213 DNA barcode was divided by the total number of lectin barcodes and expressed as percentage

$214(\%)$ values for each lectin. The microbiotas obtained from the young and old mice were also

215 analyzed by $16 \mathrm{~S}$ rRNA gene sequencing to identify the populations of bacteria.

\section{Glycan-seq of the cultured bacteria}

217 We first evaluated whether Glycan-seq can be used to profile the glycans of cultured gram-

218 positive D. radiodurans and gram-negative E. coli (Fig. 2, Table S2). Bacterial cells $\left(1 \times 10^{7}\right)$

219 were incubated with DNA-barcoded lectins, and the DNA barcodes that were released from $1 \times$

$22010^{6}$ bacterial cells by UV irradiation were counted by sequencing. The resulting lectin binding

221 signals were first analyzed by the hierarchical cluster analysis (Fig. 2A). The two types of 
222 bacteria were clearly separated into two different clusters, where the $x$-axis shows the lectins

223 used and the $y$-axis shows the bacterial species. Several lectins differentially bound to $D$.

224 radiodurans and E. coli. Specifically, mannose-binders (rGRFT and rBanana) reacted at

225 significantly higher levels to D. radiodurans $(p<0.01)$, whereas GalNAc (HPA)-, Gal $\beta 1$ -

226 3GlcNAc/GlcNAc (rABA, rSRL)-, GlcNAc (rPVL)-, and rhamnose (CSA)-binders exclusively

227 reacted with D. radiodurans ( $p<0.01$, Fig. 2B).

228 We validated the results of lectin binding to D. radiodurans and E. coli obtained by Glycan-seq

229 using flow cytometry analysis, which is considered the gold standard. Lectins that specifically

230 bound to the two different types of bacteria were identified by $t$-test analysis (Table 1). Sixteen

231 lectins specifically bound at significant levels $(p<0.01$, Fig $2 \mathrm{~B})$. Based on the signal intensity

232 (average intensity for positive cells, $>0.5$ ) obtained from Glycan-seq and the $t$-ratio $(>20)$ from

233 statistical analysis, we selected the following four lectins for flow cytometry analysis (Fig. 2B

234 and Table 1): mannose-binders (rGRFT, rBanana) that generated higher signals in $D$.

235 radiodurans and Gal $31-3 \mathrm{GalNAc/GlcNAc-binders} \mathrm{(rABA,} \mathrm{rSRL)} \mathrm{that} \mathrm{generated} \mathrm{higher} \mathrm{signals}$

236 in E. coli.

237 Flow cytometry using fluorescently labeled mannose-binders (rGRFT, rBanana) generated a

238 higher peak signal in D. radiodurans, whereas similar analysis using Gal $\beta 1-3$ GalNAc/GlcNAc- 
239 binders (rABA, rSRL) generated a higher peak signal in E. coli (Fig. 2C). Thus, the results of

240 flow cytometry agreed with those obtained by Glycan-seq (Fig. 2B). Taken together, these results

241 indicate that bacterial glycan profiles generated by Glycan-seq can be recapitulated by flow

242 cytometry analysis.

\section{Glycan-seq of the gut microbiota from young and old mice}

244 As Glycan-seq was applicable for both gram-positive and gram-negative bacteria, we used this

245 approach to profile the gut microbiota. Bacterial cells were from the fecal microbiota of young,

246 preweaned (14-20 days) and old (12 months) mice $(n=3)$, and the numbers and sizes of cells are

247 shown in Table 2. On average, the bacterial cells from the feces of young mice numbered $1.6 \mathrm{x}$

$24810^{10}$ cells/g with an average diameter of $1.2 \mu \mathrm{m}$, whereas those from old mice numbered $1.8 \mathrm{x}$

$24910^{10}$ cells/g with an average diameter of $0.97 \mu \mathrm{m}$. The fecal microbiotas $\left(1 \mathrm{x} 10^{7}\right.$ cells $)$ of young

250 and old mice were then subjected to Glycan-seq, followed by hierarchical cluster analysis. Figure

$2513 \mathrm{~A}$ shows that the gut microbiotas of young and old mice are separated into two clusters based

252 on Glycan-seq (Table S3), demonstrating that the gut microbiotas of young and old mice have

253 distinct glycan profiles. Statistical analysis on the lectin intensity data obtained from Glycan-seq

254 reveals four lectins that significantly differentiated the young and old microbiotas (Table 3).

255 These lectins are $\alpha 2-6$ Sia-binders (SSA, TJAI) and Gal $\beta 1-3$ GalNAc/GlcNAc-binders (rSRL, 
256 rABA) (Fig. 3B), all of which were detected at higher levels in the young microbiota. Previous

257 studies have shown that the composition and diversity of the gut microbiota change with age

258 [24]. Notably, the levels of some lectins, including rhamnose (CSA) and fucose-binders (rPhoSL,

$259 \mathrm{rBC} 2 \mathrm{LCN}$ ) (Fig. 3B), tend to be higher in old microbiota. However, the difference is not

260 statistically significant (Fig. 3B), which may be due to the increases in microbial diversity and

261 mouse-to-mouse variation in the composition of microbiota in old mice. Nevertheless, our data

262 suggest that our newly developed Glycan-seq technology successfully profiled the glycome of

263 the gut microbiota.

264 Differences in the composition of the gut microbiota of young and old mice

265 Based on 16S rRNA sequencing, the $\alpha$ - and $\beta$-diversity of young and old gut microbiota differed

266 (Figs. 4A and 4B). This result is similar to that of a previous study [25] that reported differing

267 compositions of the gut microbiota of young and old mice. The analysis of the ASVs of the

268 metagenomic data of 16S rRNA gene sequences using QIIME2 [18] shows family-level

269 differences between young and old microbiotas. Specifically, the relative abundance levels of

270 Lactobacillaceae, Enterobacteriaceae, Pseudomonadaceae, and Gemellaceae were higher in

271 young microbiota. In contrast, Rikenellaceae, Erysipelotrichaceae, Muribaculaceae,

272 Bifidobacteriaceae, and Lachnospiraceae were higher in old microbiota (Fig. 4C). Therefore, 
273 these differences in gut microbiota diversity may explain the different glycan profiles of young

274 and old microbiotas, as revealed by Glycan-seq analysis.

275 Changes in gut microbiota diversity are associated with the different glycans detected in

\section{6 the gut microbiota}

277 We investigated whether the differences in the relative abundance levels of bacterial families are

278 correlated with the lectins identified by Glycan-seq analysis through Spearman's correlation

279 analysis. The analysis revealed the correlation between the relative abundance values of the

280 bacteria and the lectin signal intensities of the young and old microbiotas. Lectins with variable

281 correlations to the bacterial abundance were also identified (Fig. 5). $\alpha 2-6$ Sia-binders (SSA,

282 TJAI) and Galß1-3GalNAc/GlcNAc-binders (rSRL, rABA) had significantly higher intensities in

283 the young microbiota (Fig. 3). Moreover, the signal intensities of $\alpha 2-6$ Sia-binders (SSA, TJAI)

284 were positively correlated with those of Galß1-3GalNAc/GlcNAc-binders (rSRL, rABA)(Fig. 5).

285 These lectins were positively correlated with levels of Lactobacillaceae, Enterobacteriaceae, and

286 Gemilaceae but were negatively correlated with the Muribaculaceae, Lachnospiraceae,

287 Erysipelotrichaceae, and Rikenellaceae.

288 Identification of sialylated bacteria in the gut microbiota of young mice 
289 The signal intensities of $\alpha 2-6$ Sia-binders (SSA, TJAI) were significantly higher in the microbiota

290 of young mice. We were thus interested in investigating which bacteria are covered with Sia, a

291 monosaccharide that is primarily found at the nonreducing end of glycoconjugates in eukaryotes

292 and is involved in a variety of cell-cell interactions and cell-molecule recognition [26]. Several

293 species of pathogenic bacteria display Sia on their outer surfaces, which masks them from the

294 host immune system [27]. We used a lectin pull-down assay to determine which bacteria in the

295 microbiota of young mice react with a2-6Sia-binders. The assay involved the incubation of

296 bacterial cells (isolated from young and old mice) together with magnetic beads coated with $\alpha 2-$

297 6Sia-binders (SSA, TJAI). The average number of cells recovered from the young microbiota by

298 the assay was $2.5 \times 10^{6}$ cells/g, with an average diameter of $1.1 \mu \mathrm{m}$, whereas only approximately

299 one-hundredth of bacterial cells (an average of $1.9 \times 10^{4}$ cells/g with an average diameter of

$300 \quad 0.6 \mu \mathrm{m}$ ) were obtained in the old mice (Table 4). The incubation of both types of microbiotas

301 with lectin-coated beads resulted in the recovery of more cells from the young microbiota,

302 indicating that more bacterial cells with glycans that react with $\alpha 2-6$ Sia-binders are present in

303 young mice (Fig. 6).

304 The identification of the taxonomic groups of bacteria pulled down by the $\alpha 2-6$ Sia-binders is

305 based on their 16S rRNA gene sequences. The ASV analysis (using QIIME2) of the 16S rRNA

306 gene sequences from the metagenomic data shows the phylogenetic distribution and relative 
307 abundance of bacteria pulled down by SSA and TJAI. The results identify the families of bacteria

308 present at higher levels in the young microbiota (Fig. 6). Both lectins detected mainly

309 Lactobacillaceae, Lachnospiraceae, Enterobacteriaceae, and Muribaculaceae (Fig. 6). At the

310 genus level, these bacteria consisted mainly of Lactobacillus, Pseudomonas, Escherichia-

311 Shigella, and Streptococcus. Therefore, these bacterial families and genera are likely modified by

312 sialylated glycans. These results show that the bacteria recovered from the gut microbiota using

313 lectins are those covered by Sia.

\section{Discussion}

316 The genome and metabolome are the two major omics data acquired for the microbiota. In this

317 sense, the information of the intact bacterial cell surface molecules without prior in vitro

318 culturing, which directly mediate microbe-host interactions, coundn't be acquired. Actually,

319 bacterial cell surfaces are coated with a diverse array of glycans that play pivotal roles in various

320 biological processes. In particular, they mediate microbe-host interactions during the onset and

321 development of infectious diseases and symbiotic interactions. However, our understanding of

322 the glycome of the gut microbiota remains limited because of the lack of appropriate methods of

323 analysis. In this study, we have developed a new sequencing-based technology that can analyze 
324 the glycome of bacteria in an intact form. The Glycan-seq technology offers several advantages:

325 (1) Live bacterial cells can be analyzed without the need for prior culturing. (2) Fluorescence

326 labeling of bacteria is not required. (3) A relatively low number of bacterial cells $\left(\sim 10^{6}\right.$ cells $)$ are

327 required for the analysis. (4) Glycomic profiles can be acquired using a conventional next-

328 generation sequencer, the same instrument used for 16S rRNA gene sequencing.

329 The diversity of the gut microbiota of old mice differed from that of young mice. The relative

330 abundance levels of Lactobacillaceae, Enterobacteriaceae, Pseudomonadaceae, and

331 Gemellaceae were higher in young than in old microbiota, whereas those of Rikenellaceae,

332 Erysipelotrichaceae, Muribaculaceae, Bifidobacteriaceae, and Lachnospiraceae were higher in

333 old than in young microbiota. These differences in the gut microbiota diversity may be due to

334 host differences in feeding habits; young mice are nursed on mother's milk, whereas old mice are

335 fed with normal chow diets. In humans, breastfeeding babies have more Lactobacilli in their gut

336 microbiota than those of milk formula-fed babies [28]. These findings and the results of this

337 study (Fig. 4) suggest the common presence of Lactobacilli in the gut of breastfed animals. A

338 study on calorie-restricted mice found reduced levels of Lactobacillus, which was negatively

339 correlated with mice lifespan [29]. Furthermore, the abundance of Lactobacillus murinus is

340 higher in calorie-restricted mice, and this species promoted anti-inflammation, which may play

341 an important role during aging [30]. 
342 Several studies have sought to understand how the gut microbiota changes during aging and the

343 biological significance of such changes [31]. The results from longitudinal studies on fecal

344 samples from various individuals of different ages show age-related changes in the diversity and

345 composition of the human gut microbiota $[32,33]$. The composition of the gut microbiota of

346 older adults is unique, and the $\alpha$-diversity of this microbiota increases with age, suggesting a

347 correlation between the composition of the gut microbiota and physiological aging [33].

348 In this study, we report for the first time that the glycome of the gut microbiota changes during

349 aging. Glycan-seq technology was able to profile the microbial glycomes of young and old mice,

350 and interestingly, $\alpha 2-6$ Sia-binders reacted at significantly higher levels with the young

351 microbiota, suggesting that the levels of sialylated bacteria decrease during aging. The bacterial

352 families that reacted most with a2-6Sia-binders are Lactobacillaceae, Lachnospiraceae,

353 Enterobacteriaceae, and Muribaculaceae. Previous findings that some Lactobacillus species

354 express genes involved in the catabolism of Sia [27] are consistent with our results. The presence

355 of Sia in Lactobacillus species has also been previously reported [34]. Several pathogenic

356 bacteria such as enterohemorrhagic Escherichia coli, Haemophilus influenzae, H. ducreyi,

357 Pasteurella multocida, Neisseria gonorrhoeae, N. meningitidis, Campylobacter jejuni, and

358 Streptococcus agalactiae are well known to display Sia on their outer surfaces, which masks

359 them from the host immune system. These bacteria have developed different mechanisms for 
360 obtaining Sia, including the de novo biosynthesis of Sia (E. coli, N. meningitidis), Sia scavenging

361 (N. gonorrhoeae), and precursor scavenging (H. influenzae) [27]. One of the functions of Sia is

362 the regulation of innate immunity by providing a mechanism of identifying self from nonself.

363 However, various microbes have evolved a counter-mechanism that works by decorating the

364 bacterial cell surface with similar Sia modifications [35]. Sia that decorates the bacterial surface

365 regulates the host immune system by interacting with sialic acid-binding immunoglobulin-type

366 lectins (Siglecs) [36]. The presence of the Sia on the surface of bacteria also protects them

367 against invading bacteriophage by blocking the relevant underlying receptors [37]. Therefore, the

368 presence of Sia on the surface of bacteria in the gut microbiota of young mice suggests that these

369 microbes are protected from the host's innate immune surveillance system and from

370 bacteriophage, and their establishment proceeds from an initial colonization by microbes in the

371 gut of young mice [35].

372 Most bacteria obtain Sia by scavenging it from the surrounding environment [27]. Therefore, the

373 glycans of bacteria cultured in vitro most probably differs from that of the same bacteria growing

374 in the gut. Given this situation, Glycan-seq is useful because it can capture the glycomic

375 information of gut bacteria in situ, because it does not require prior culturing in vitro.

376 The following are the limitations of the current method: (1) the glycomic profile of single 
377 bacterial cells cannot be obtained; and (2) the method is unable to determine the detailed

378 structure of glycans. We aim to solve the first limitation by developing a method of

379 simultaneously analyzing the glycome and genome of single bacterial cells.

\section{Conclusions}

382 Our results suggest that the Glycan-seq method is an excellent choice for profiling the glycome

383 of the gut microbiota. Our data provides important (and previously unknown) details about the

384 changes in the glycome of the gut microbiota during aging. Glycan-seq analysis, in parallel with

385 16S rRNA gene sequencing, can identify the bacteria modified with Sia. It will be interesting to

386 apply the Glycan-seq technology in future studies, seeking to understand how the glycome of the

387 gut microbiota changes in response to dietary changes and disease development. Moreover,

388 application of the Glycan-seq method to profile the glycome of a single bacterial cell, along with

389 the bacterial identification, will help researchers understand the glycome architecture of the gut

390 microbiota and its interaction with the host. In addition, our technology can also be applied to

391 profile the glycomes of other bacterial communities, such as those in the soil, deep ocean, and

392 volcanic deposits. 


\section{Acknowledgments}

395 The authors would like to thank Professor Hiromi Yanagisawa and the Animal Resource Center

396 (ARC) staff of the University of Tsukuba for assisting in collecting the mouse feces; Drs. Jonguk

397 Park and Jun Kunisawa at National Institutes of Biomedical Innovation, Health and Nutrition,

398 and Drs. Hiroyuki Kusada and Hideyuki Tamaki at the National Institutes of Advanced Industrial

399 and Technology for technical advice on 16S rRNA sequencing; Ms. Sunanda Keisham for her

400 helpful discussion during the experiment.

402 Authors' contributions

403 LO performed experiments and data analysis and wrote the paper. FM performed experiments

404 and data analysis. HT designed this study, led this study, and wrote the paper. All authors

405 provided feedback and contributed to the research and final manuscript.

407 Funding

408 This research was supported by AMED-Prime, AMED, under grant number 21 gm6010018h0004

409 and Yakult Bio-Science Foundation. 


\section{Availability of data and materials}

412 All the data generated and analyzed have been included in the article or as supplementary tables

413 and files. The raw $16 \mathrm{~S}$ rRNA amplicon sequencing data are deposited and publicly available

414 from European Nucleotide Archive (ENA) at EMBL-EBI under accession number PRJEB45936

415 (https://www.ebi.ac.uk/ena/browser/view/PRJEB45936).

416

\section{Declarations}

\section{Ethics approval and consent to participate}

419 Not applicable

\section{Consent for publication}

$421 \quad$ Not applicable

\section{Competing interests}

423 The authors declare that they have no competing interests. 


\section{References}

426 1. Costello EK, Lauber CL, Hamady M, Fierer N, Gordon JI, Knight R. Bacterial Community

427 Variation in human body habitats across space and time. Science (80- ). 2009;326:1694 LP -

428 1697. doi:10.1126/science.1177486.

429 2. Qin J, Li R, Raes J, Arumugam M, Burgdorf KS, Manichanh C, et al. A human gut microbial

430 gene catalogue established by metagenomic sequencing. Nature. 2010;464:59-65.

431 doi:10.1038/nature08821.

432 3. Eckburg PB, Bik EM, Bernstein CN, Purdom E, Dethlefsen L, Sargent M, et al. Diversity of

433 the human intestinal microbial flora. Science (80- ). 2005;308:1635 LP - 1638.

434 doi:10.1126/science.1110591.

435 4. Valdes AM, Walter J, Segal E, Spector TD. Role of the gut microbiota in nutrition and health.

436 BMJ. 2018;361:k2179. doi:10.1136/bmj.k2179.

437 5. Kayama H, Okumura R, Takeda K. Interaction between the microbiota, epithelia, and immune

438 cells in the intestine. Annu Rev Immunol. 2020;38:23-48. doi:10.1146/annurev-immunol-

439 070119-115104. 
440 6. Carabotti M, Scirocco A, Maselli MA, Severi C. The gut-brain axis: interactions between

441 enteric microbiota, central and enteric nervous systems. Ann Gastroenterol. 2015;28:203-9.

442 https://pubmed.ncbi.nlm.nih.gov/25830558.

443 7. Sekirov I, Russell SL, Antunes LCM, Finlay BB. Gut microbiota in health and disease.

444 Physiol Rev. 2010;90:859-904. doi:10.1152/physrev.00045.2009.

445 8. Boulangé CL, Neves AL, Chilloux J, Nicholson JK, Dumas M-E. Impact of the gut microbiota

446 on inflammation, obesity, and metabolic disease. Genome Med. 2016;8:42. doi:10.1186/s13073-

447 016-0303-2.

448 9. Kamada N, Seo S-U, Chen GY, Núñez G. Role of the gut microbiota in immunity and

449 inflammatory disease. Nat Rev Immunol. 2013;13:321-35. doi:10.1038/nri3430.

450 10. Mescher MF, Strominger JL, Watson SW. Protein and carbohydrate composition of the cell

451 envelope of Halobacterium salinarium. J Bacteriol. 1974;120:945-54.

452 11. Silhavy TJ, Kahne D, Walker S. The bacterial cell envelope. Cold Spring Harb Perspect Biol.

453 2010;2:a000414-a000414. doi:10.1101/cshperspect.a000414.

454 12. Beveridge TJ. Structures of gram-negative cell walls and their derived membrane vesicles. J

455 Bacteriol. 1999;181:4725-33. doi:10.1128/JB.181.16.4725-4733.1999. 
456 13. Whitfield C. Bacterial extracellular polysaccharides. Can J Microbiol. 1988;34:415-20.

457 doi: $10.1139 / \mathrm{m} 88-073$.

458 14. Emi Y, Hiroaki T, Jun H, Tohru I, Tomoyuki S. Lectin microarray reveals binding profiles of

459 Lactobacillus casei strains in a comprehensive analysis of bacterial cell wall polysaccharides.

460 Appl Environ Microbiol. 2011;77:4539-46. doi:10.1128/AEM.00240-11.

461 15. Minoshima F, Ozaki H, Odaka H, Tateno H. Integrated analysis of glycan and RNA in single

462 cells. bioRxiv. 2021;:2020.06.15.153536. doi:10.1101/2020.06.15.153536.

463 16. Hevia A, Delgado S, Margolles A, Sánchez B. Application of density gradient for the

464 isolation of the fecal microbial stool component and the potential use thereof. Sci Rep.

465 2015;5:16807. doi:10.1038/srep16807.

466 17. Klindworth A, Pruesse E, Schweer T, Peplies J, Quast C, Horn M, et al. Evaluation of general

467 16S ribosomal RNA gene PCR primers for classical and next-generation sequencing-based

468 diversity studies. Nucleic Acids Res. 2013;41:e1-e1. doi:10.1093/nar/gks808.

469 18. Bolyen E, Rideout JR, Dillon MR, Bokulich NA, Abnet CC, Al-Ghalith GA, et al.

470 Reproducible, interactive, scalable and extensible microbiome data science using QIIME 2. Nat

471 Biotechnol. 2019;37:852-7. doi:10.1038/s41587-019-0209-9. 
472 19. Callahan BJ, McMurdie PJ, Rosen MJ, Han AW, Johnson AJA, Holmes SP. DADA2: High-

473 resolution sample inference from Illumina amplicon data. Nat Methods. 2016;13:581-3.

474 doi:10.1038/nmeth.3869.

475 20. Bokulich NA, Kaehler BD, Rideout JR, Dillon M, Bolyen E, Knight R, et al. Optimizing

476 taxonomic classification of marker-gene amplicon sequences with QIIME 2's q2-feature-

477 classifier plugin. Microbiome. 2018;6:90. doi:10.1186/s40168-018-0470-z.

478 21. Quast C, Pruesse E, Yilmaz P, Gerken J, Schweer T, Yarza P, et al. The SILVA ribosomal

479 RNA gene database project: Improved data processing and web-based tools. Nucleic Acids Res.

480 2013;41 Database issue:D590-6. doi:10.1093/nar/gks1219.

481 22. Robeson M, O’Rourke D, Kaehler B, Ziemski M, Dillon M, Foster J, et al. RESCRIPt:

482 Reproducible sequence taxonomy reference database management for the masses. 2020.

483 doi:10.1101/2020.10.05.326504.

484 23. Catherine L, Rob K. UniFrac: a New Phylogenetic Method for Comparing Microbial

485 Communities. Appl Environ Microbiol. 2005;71:8228-35. doi:10.1128/AEM.71.12.8228-

$486 \quad 8235.2005$.

487 24. Claesson MJ, Cusack S, O’Sullivan O, Greene-Diniz R, de Weerd H, Flannery E, et al. 
488 Composition, variability, and temporal stability of the intestinal microbiota of the elderly. Proc

489 Natl Acad Sci U S A. 2011;108 Suppl Suppl 1:4586-91. doi:10.1073/pnas.1000097107.

490 25. Low A, Soh M, Miyake S, Seedorf H. Host-age prediction from fecal microbiome

491 composition in laboratory mice. bioRxiv. 2020;:2020.12.04.412734.

492 doi:10.1101/2020.12.04.412734.

493 26. Varki A. Sialic acids in human health and disease. Trends Mol Med. 2008;14:351-60.

494 doi:10.1016/j.molmed.2008.06.002.

495 27. Almagro-Moreno S, Boyd EF. Insights into the evolution of sialic acid catabolism among

496 bacteria. BMC Evol Biol. 2009;9:118. doi:10.1186/1471-2148-9-118.

497 28. Bäckhed F, Roswall J, Peng Y, Feng Q, Jia H, Kovatcheva-Datchary P, et al. Dynamics and

498 stabilization of the human gut microbiome during the first year of life. Cell Host Microbe.

499 2015;17:852. doi:https://doi.org/10.1016/j.chom.2015.05.012.

500 29. Zhang C, Li S, Yang L, Huang P, Li W, Wang S, et al. Structural modulation of gut

501 microbiota in life-long calorie-restricted mice. Nat Commun. 2013;4:2163.

502 doi:10.1038/ncomms3163.

503 30. Pan F, Zhang L, Li M, Hu Y, Zeng B, Yuan H, et al. Predominant gut Lactobacillus murinus 
504 strain mediates anti-inflammaging effects in calorie-restricted mice. Microbiome. 2018;6:54.

505 doi:10.1186/s40168-018-0440-5.

506 31. Yatsunenko T, Rey FE, Manary MJ, Trehan I, Dominguez-Bello MG, Contreras M, et al.

507 Human gut microbiome viewed across age and geography. Nature. 2012;486:222-7.

508 doi:10.1038/nature11053.

509 32. Claesson MJ, Jeffery IB, Conde S, Power SE, O’Connor EM, Cusack S, et al. Gut microbiota

510 composition correlates with diet and health in the elderly. Nature. 2012;488:178-84.

511 doi:10.1038/nature11319.

512 33. van Tongeren SP, Slaets JPJ, Harmsen HJM, Welling GW. Fecal microbiota composition and

513 frailty. Appl Environ Microbiol. 2005;71:6438-42. doi:10.1128/AEM.71.10.6438-6442.2005.

514 34. Sakellaris G, Kolisis FN, Evangelopoulos AE. Presence of sialic acids in Lactobacillus

515 plantarum. Biochem Biophys Res Commun. 1988;155:1126-32.

516 doi:https://doi.org/10.1016/S0006-291X(88)81257-9.

517 35. Varki A, Gagneux P. Multifarious roles of sialic acids in immunity. Ann N Y Acad Sci.

518 2012;1253:16-36. doi:10.1111/j.1749-6632.2012.06517.x.

519 36. Chang Y-C, Nizet V. Siglecs at the host-pathogen interface. Adv Exp Med Biol. 
520 2020;1204:197-214. doi:10.1007/978-981-15-1580-4_8.

521 37. Angata T, Varki A. Chemical diversity in the sialic acids and related $\alpha$-keto acids: An

522 evolutionary perspective. Chem Rev. 2002;102:439-70. doi:10.1021/cr000407m.

\section{Figure Legends}

525 Fig. 1. Glycomic profiling of microbiota by Glycan-seq. (A) Schematic representation of a DNA-

526 barcoded lectin. (B) Experimental workflow of the Glycan-seq and 16S rRNA sequencing of

527 microbiota. (C) Schematic representation of lectin pull-down followed by 16S rRNA sequencing

528 to identify lectin-reactive bacteria.

529 Fig. 2. Glycomic profiling of the cultured bacteria by Glycan-seq. (A) Hierarchical cluster

530 analysis of D. radiodurans and E. coli using Glycan-seq data. (B) Graphical representation of

531 Glycan-seq intensity data of D. radiodurans and E. coli. (C) Comparison between Glycan-seq

532 (top panel) and flow cytometry data (bottom panel). Blue: D. radiodurans; red: E. coli.

533 Fig. 3. Glycomic profiling of the gut microbiota of young and old mice. (A) Hierarchical

534 clustering analysis of the gut microbiota of young and old mice using Glycan-seq data. (B)

535 Graphical representation of the Glycan-seq data of the gut microbiota of young and old mice. 
536 Fig. 4. Comparison of the bacterial abundance between the young and old mice microbiota. (A)

537 Boxplot of Faith's phylogenetic diversity (PD) metrics analysis for the $\alpha$-diversity. Statistical

538 significance $(p<0.05)$ is denoted with an asterisk $(*)$ calculated by Kruskal-Wallis pairwise

539 analysis. (B) $\beta$-diversity analysis by principal coordinates analysis (PCoA) of unweighted

540 UniFrac distance. (C) Stacked bar plot showing the taxonomy of the differential bacterial

541 abundance in the young and old mice microbiota obtained from each mouse by $16 \mathrm{~S}$ rRNA

542 sequencing ( $n=3$ for each age group). Each colored bar plot indicates the family of bacteria

543 identified, and for clarity, only the most abundant 11 families are shown, and the remaining are

544 shown as others

545 Fig. 5. Correlation between bacterial family and lectin. Spearman correlations between the

546 abundant bacterial family from 16S rRNA sequencing and the lectin intensity from the Glycan-

547 seq. The correlations represented were statistically significant $(p<0.05)$, and the circle's size

548 represents the strength of correlations. Red: negative; blue: positive correlations.

549 Fig. 6. Family of bacteria reactive to $\alpha 2-6 \mathrm{Sia}$ identified by lectin pull-down and $16 \mathrm{~S}$ rRNA

550 sequencing from the young microbiota. Stacked taxa bar plot represents the (A) family (B)

551 species of bacteria pull-downs by the SSA and TJAI, $\alpha 2-6$ Sia binders. Each colored bar plot

552 indicates the family or genus of bacteria identified, and for clarity, only the most abundant 
bioRxiv preprint doi: https://doi.org/10.1101/2021.06.30.450488; this version posted June 30, 2021. The copyright holder for this preprint (which was not certified by peer review) is the author/funder. All rights reserved. No reuse allowed without permission.

553 families or species are shown, and the remaining are shown as others.

554

555

556

557

558

559 
Table 1. Lectins with significantly different signals between Gram-positive and -negative bacteria selected by Glycan-seq

\begin{tabular}{|c|c|c|c|c|c|}
\hline Lectins & Rough specificity & $t$-ratio & $P$-value & $\begin{array}{l}\text { Average intensity } \\
\text { of D. radiodurans }\end{array}$ & $\begin{array}{l}\text { Average intensity } \\
\text { of E. coli }\end{array}$ \\
\hline rGRFT $^{1}$ & Man & 163.19 & 0.000000338 & 41.99 & 1.44 \\
\hline SSA & $\alpha 2-6 \mathrm{Sia}$ & 124.51 & 0.000000973 & 0.06 & 0.47 \\
\hline rMOA & $\alpha \mathrm{Gal}(\mathrm{B})$ & 88.106 & 0.00000378 & 0.03 & 0.23 \\
\hline SNA & $\alpha 2-6 \mathrm{Sia}$ & 42.021 & 0.0000709 & 0.02 & 0.13 \\
\hline rBanana & 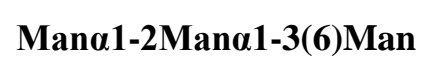 & 27.118 & 0.0003958 & 47.13 & 4.81 \\
\hline AAL & $\begin{array}{l}\alpha 1-2 F u c(H), \alpha 1-3 F u c(L e x) \\
\alpha 1-4 F u c(\text { Lea) }\end{array}$ & 26.435 & 0.0004259 & 0.31 & 4.89 \\
\hline $\mathbf{r A B A}$ & $\begin{array}{l}\text { Galß1-3GalNAc }(T), \\
\text { GlcNAc }\end{array}$ & 23.592 & 0.0006505 & 1.05 & 16.79 \\
\hline rSRL & $\begin{array}{l}\text { Galß1-3GalNAc }(T) \text {, } \\
\text { GlcNAc }\end{array}$ & 22.718 & 0.0007336 & 0.75 & 18.38 \\
\hline rRSIIL & $\begin{array}{l}\alpha 1-2 \text { Fuc (H), } \alpha 1-3 \text { Fuc (Lex), } \\
\alpha 1-3 \text { Fuc (Lea) }\end{array}$ & 21.691 & 0.0008548 & 0.11 & 3.42 \\
\hline HPA & $\alpha \operatorname{GalNAc}(\mathrm{A}, \mathrm{Tn})$ & 18.832 & 0.0014504 & 0.03 & 1.09 \\
\hline rPALa & Man5, biantenna & 17.279 & 0.001973 & 0.13 & 0.62 \\
\hline rPPL & $\begin{array}{l}\alpha, \beta \text { GalNAc }(\mathrm{A}, \mathrm{Tn}, \\
\text { LacDiNAc) }\end{array}$ & 15.275 & 0.0031018 & 0.00 & 0.01 \\
\hline rPVL & Sia, GlcNAc & 13.279 & 0.0051914 & 0.04 & 12.23 \\
\hline CSA & Rhamnose, Gala1-4Gal & 13.216 & 0.0051914 & 3.52 & 19.80 \\
\hline TJAI & $\alpha 2-6 \mathrm{Sia}$ & 12.161 & 0.0067989 & 0.01 & 0.12 \\
\hline rBC2LA & aMan, High-man & 11.036 & 0.0095384 & 0.27 & 0.15 \\
\hline
\end{tabular}

${ }^{1}$ Lectins in bold characters were selected for further flow cytometry analysis 
Table 2. Total number of bacterial cells and the average size prepared from the microbiotas of young and old mice

\begin{tabular}{lll}
\hline & Cell number from $1 \mathrm{~g}$ faces $\left(\times 10^{10}\right)$ & Average diameter size $(\mu \mathrm{m})$ \\
\hline Young 1 & 3.09 & 1.1 \\
Young 2 & 3.96 & 1.2 \\
Young 3 & 4.15 & 1.2 \\
Old 1 & 3.09 & 1 \\
Old 2 & 3.96 & 0.97 \\
Old 3 & 4.15 & 0.97 \\
\hline
\end{tabular}


Table 3. Lectins with significantly different signals between young and old microbiota selected by Glycan-seq

\begin{tabular}{l|lllll}
\hline Lectins & Rough specificity & $t$-ratio & $P$-value & $\begin{array}{l}\text { Average intensity } \\
\text { of young (\%) }\end{array}$ & $\begin{array}{l}\text { Average intensity } \\
\text { of old (\%) }\end{array}$ \\
\hline SSA & $\alpha 2-6$ Sia & 28.12 & 0.0003520 & 1.09 & 0.09 \\
rSRL & Galß1-3GalNAc (T), GlcNAc & 20.20 & 0.0012750 & 7.57 & 1.42 \\
rABA & Galß1-3GalNAc (T), GlcNAc & 16.55 & 0.0027280 & 8.21 & 1.04 \\
TJAI & $\alpha 2-6$ Sia & 8.92 & 0.0293280 & 0.21 & 0.02 \\
\hline
\end{tabular}


Table 4. Cell number and average size of bacteria pull down by SSA- and TJAI-coated beads

\begin{tabular}{|c|c|c|c|c|}
\hline & \multicolumn{2}{|l|}{ SSA pull down } & \multicolumn{2}{|l|}{ TJAI pull down } \\
\hline & $\begin{array}{l}\text { Cell number } \\
\left(\times 10^{4}\right) \text { from } 1 \mathrm{~g} \\
\text { feces }\end{array}$ & $\begin{array}{l}\text { Average diameter } \\
\text { size }(\mu \mathrm{m})\end{array}$ & $\begin{array}{l}\text { Cell number } \\
\left(\times 10^{4}\right) \text { from } 1 \mathrm{~g} \\
\text { feces }\end{array}$ & $\begin{array}{l}\text { Average diameter } \\
\text { size }(\mu \mathrm{m})\end{array}$ \\
\hline Young 1 & 263 & 1.2 & 217 & 1.1 \\
\hline Young 2 & 186 & 1.1 & 285 & 1.09 \\
\hline Young 3 & 222 & 1.09 & 286 & 1.1 \\
\hline Old 1 & 2.63 & 0.61 & 2.31 & 0.65 \\
\hline Old 2 & 1.96 & 0.58 & 2.9 & 0.6 \\
\hline Old 3 & 0.82 & 0.62 & 1.2 & 0.61 \\
\hline
\end{tabular}




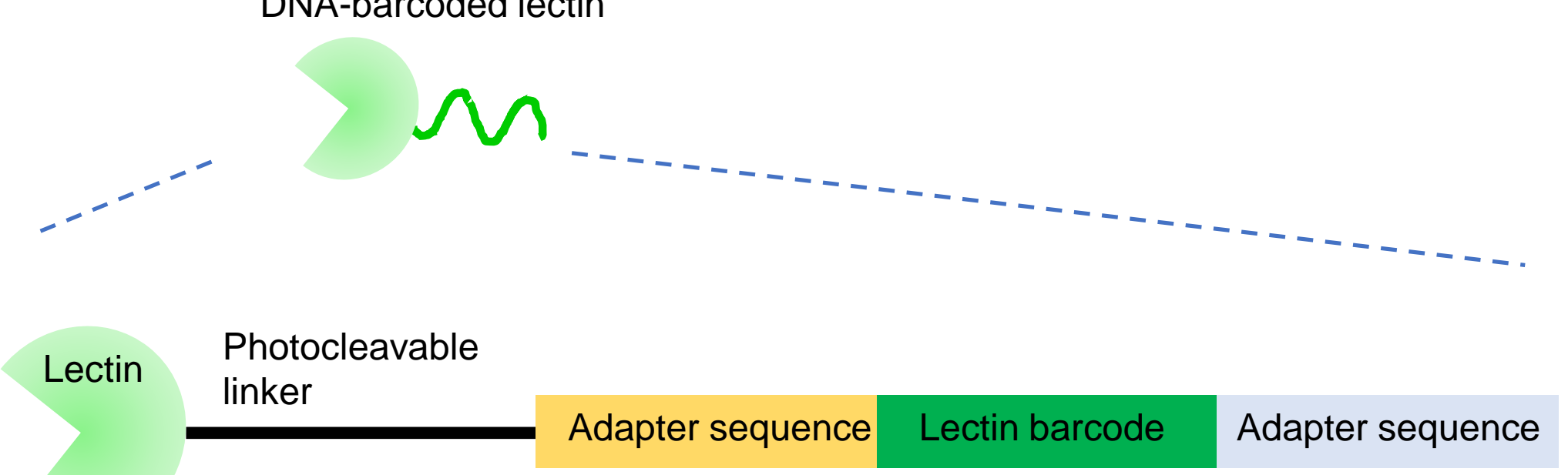

B

Microbiota DNA-barcoded lectins are reacted with bacteria

DNA barcodes

Glycan-seq are released from lectins
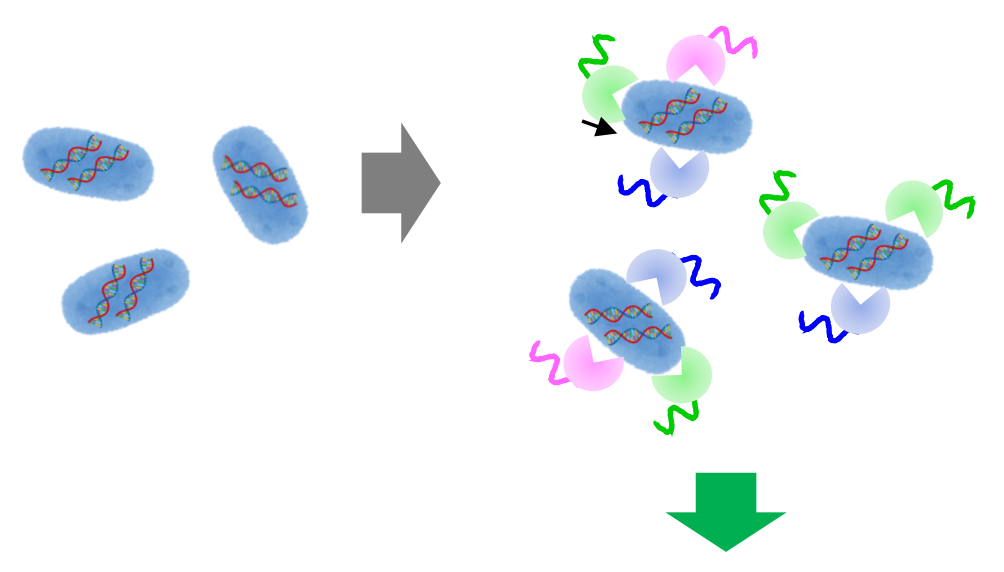

UV

3s

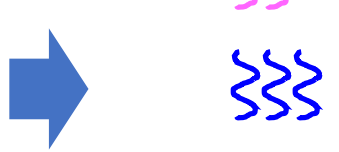

उ3S 3333

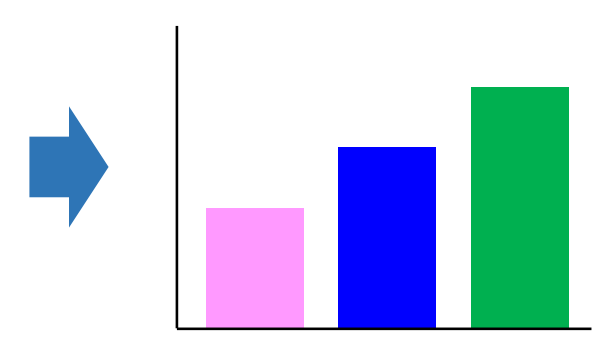

Glycomic profile
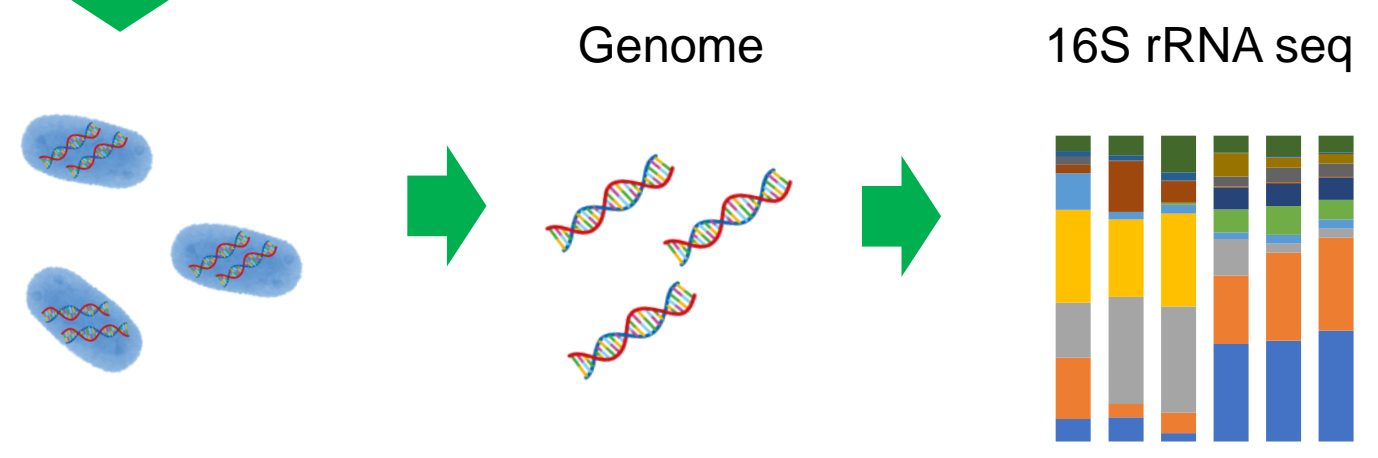

Bacterial composition

C

Microbiota

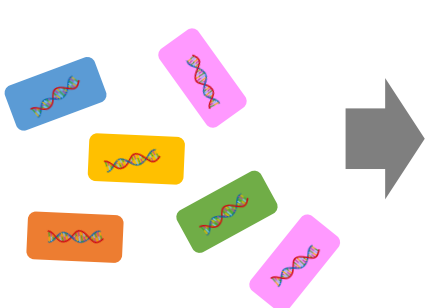

Lectin pull down using lectin-coated beads

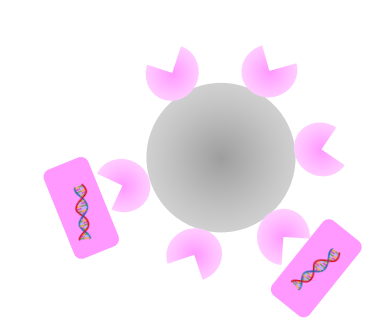

Genome

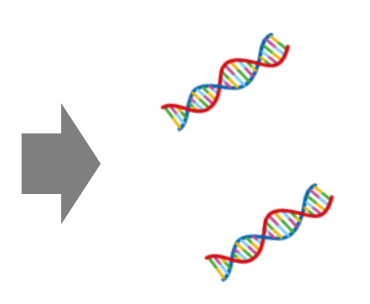

16S rRNA seq

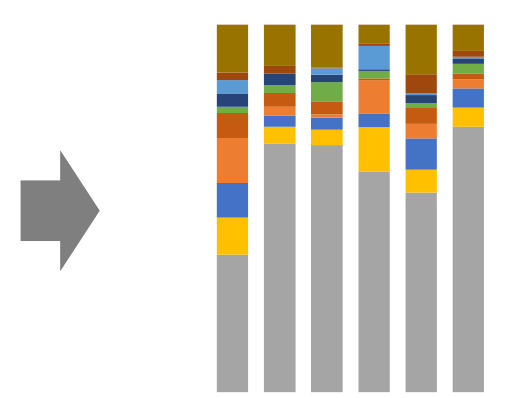

Identification of lectin-reactive bacteria 
A

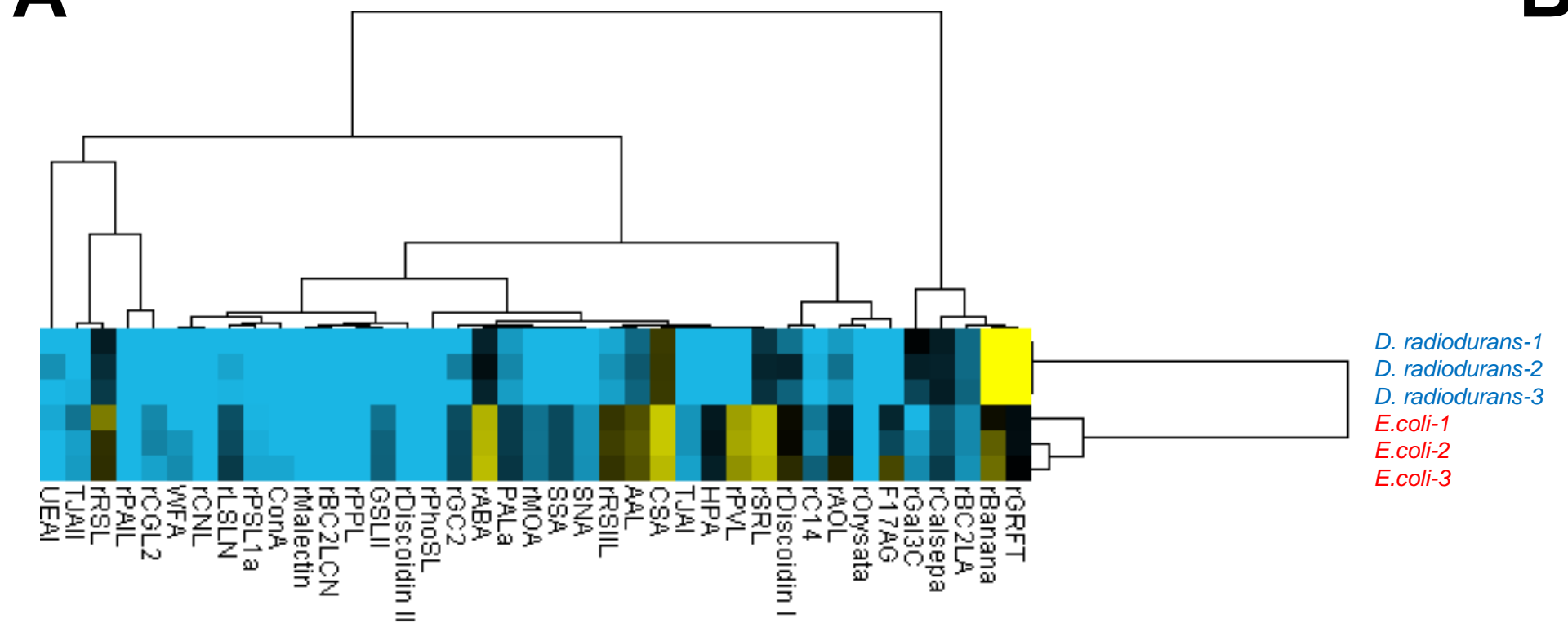

B

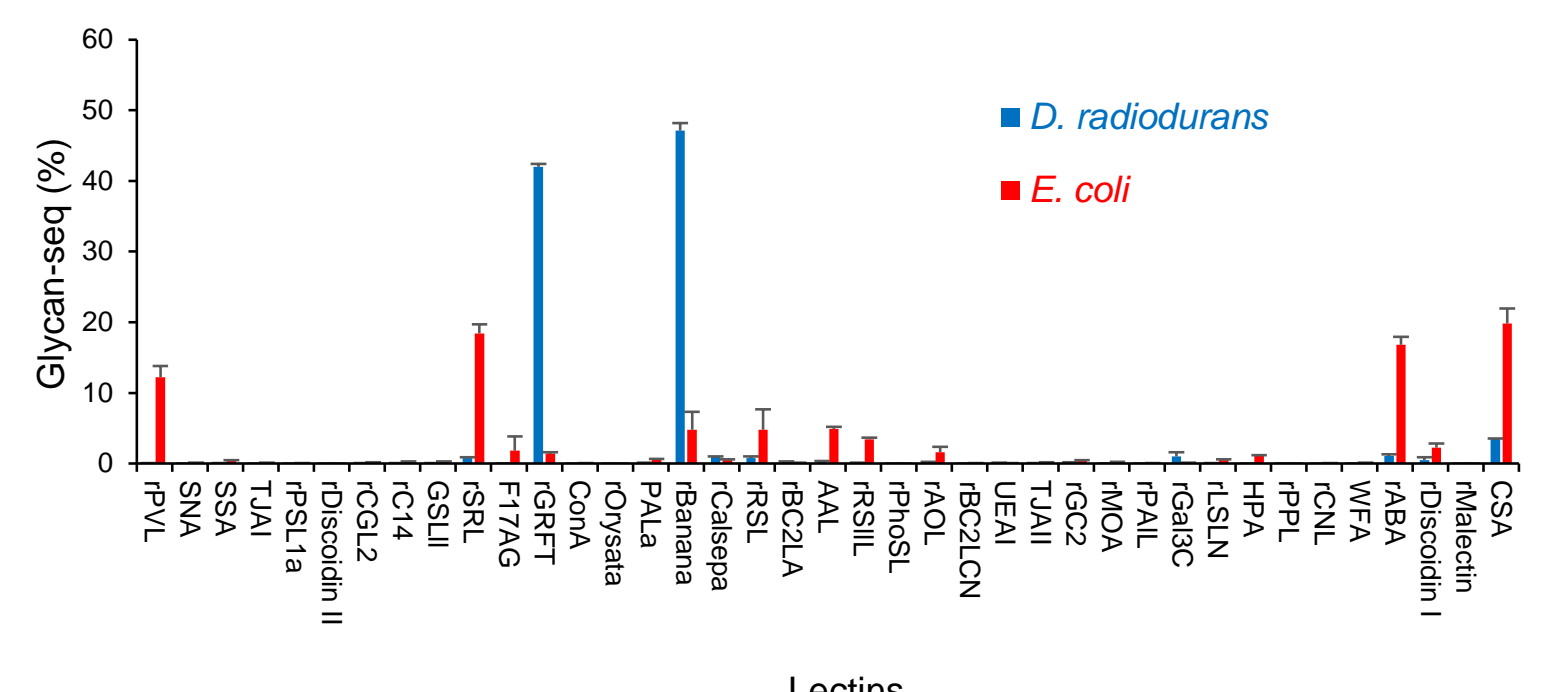

C
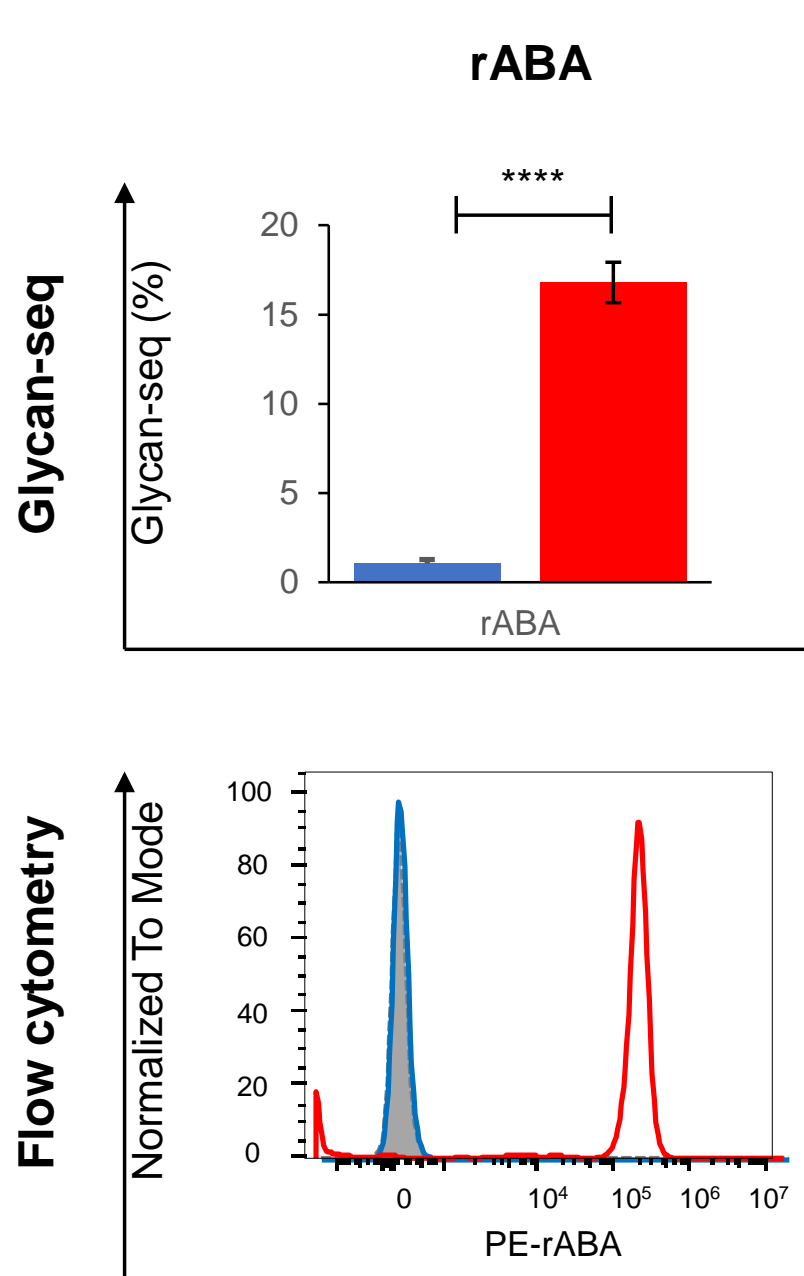

rSRL

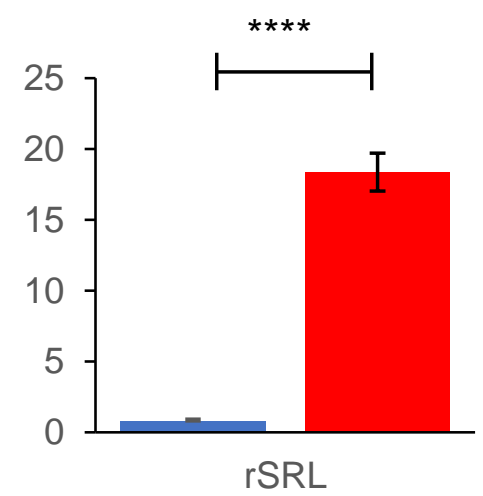

SRL

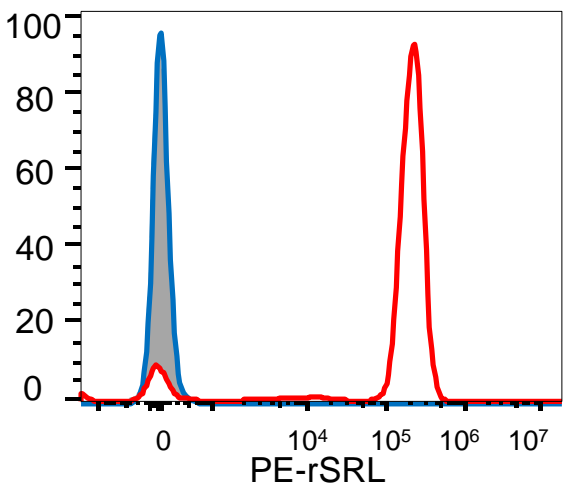

rGRFT

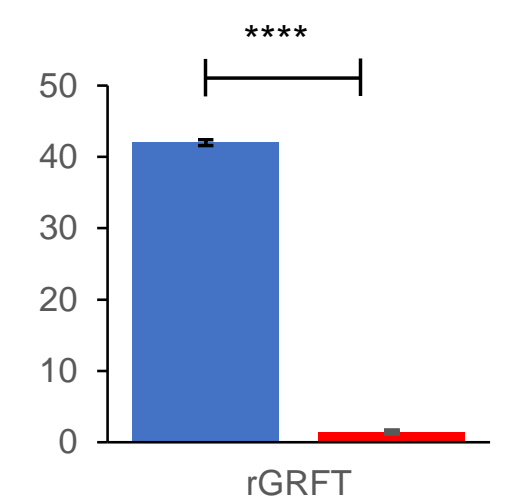

Lectin

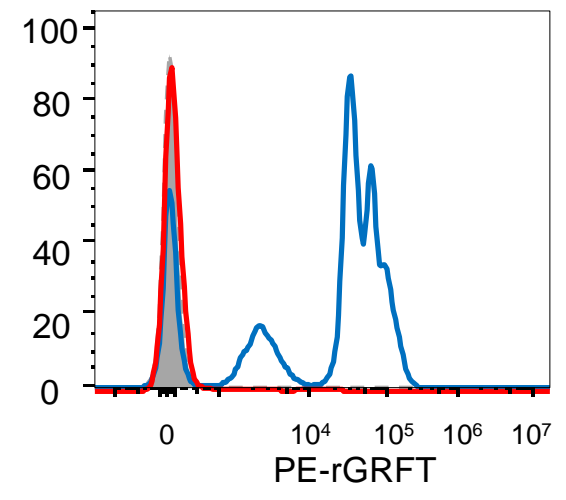

rBanana

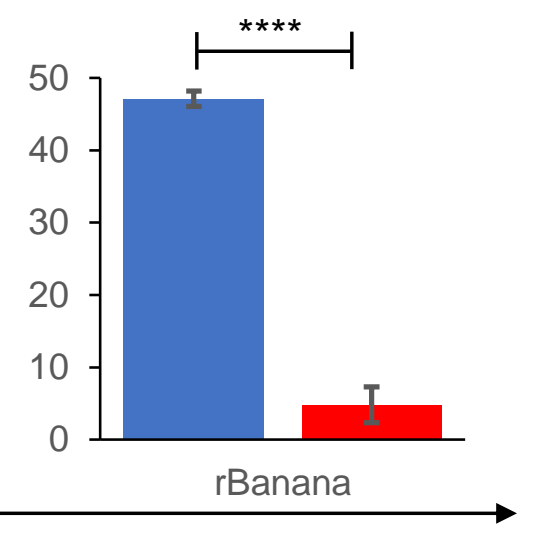

- D. radiodurans

- E. coli

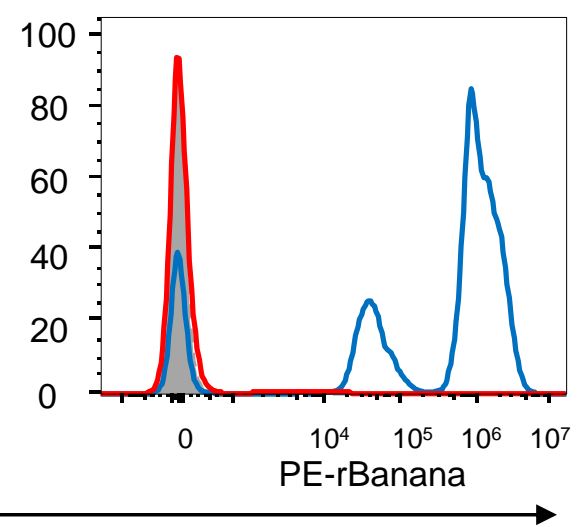

- D. radiodurans

- E. coli 
A

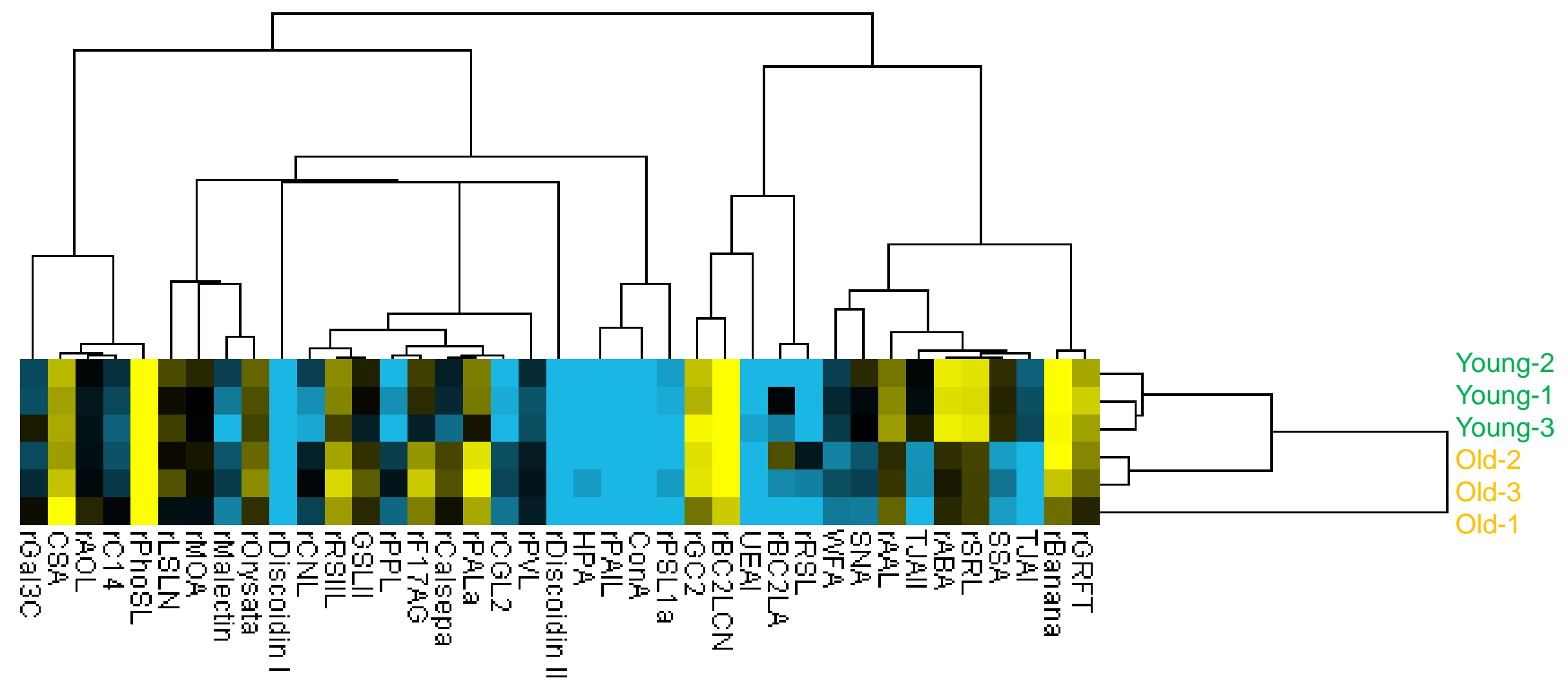

B

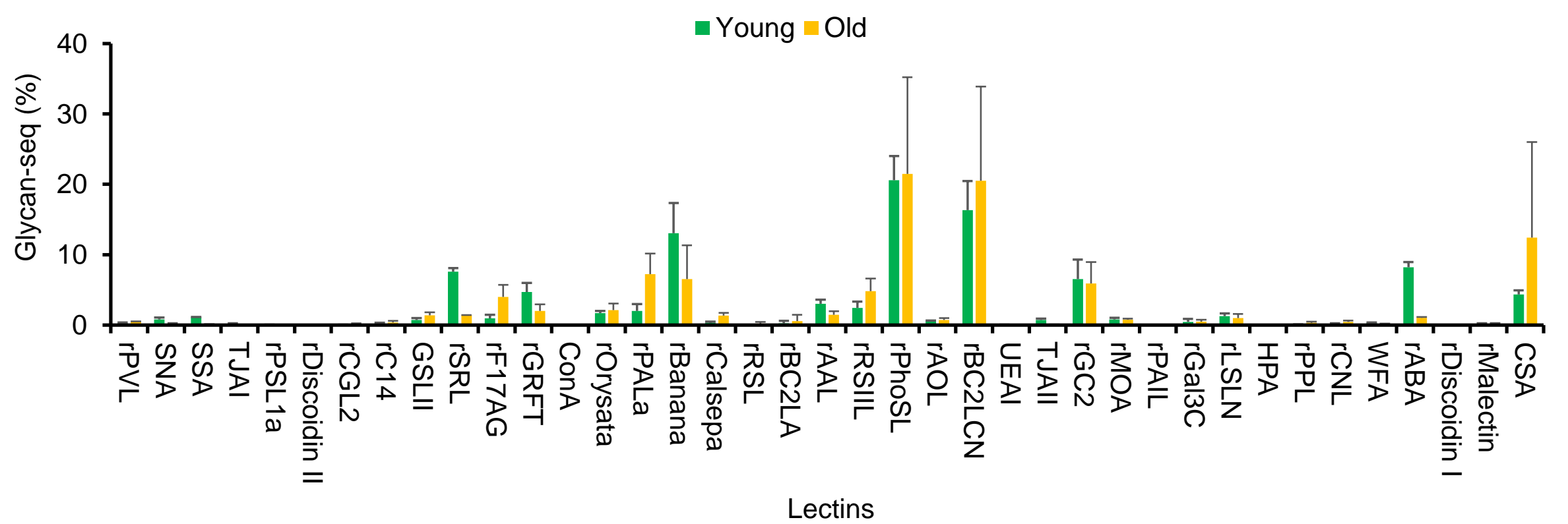


A

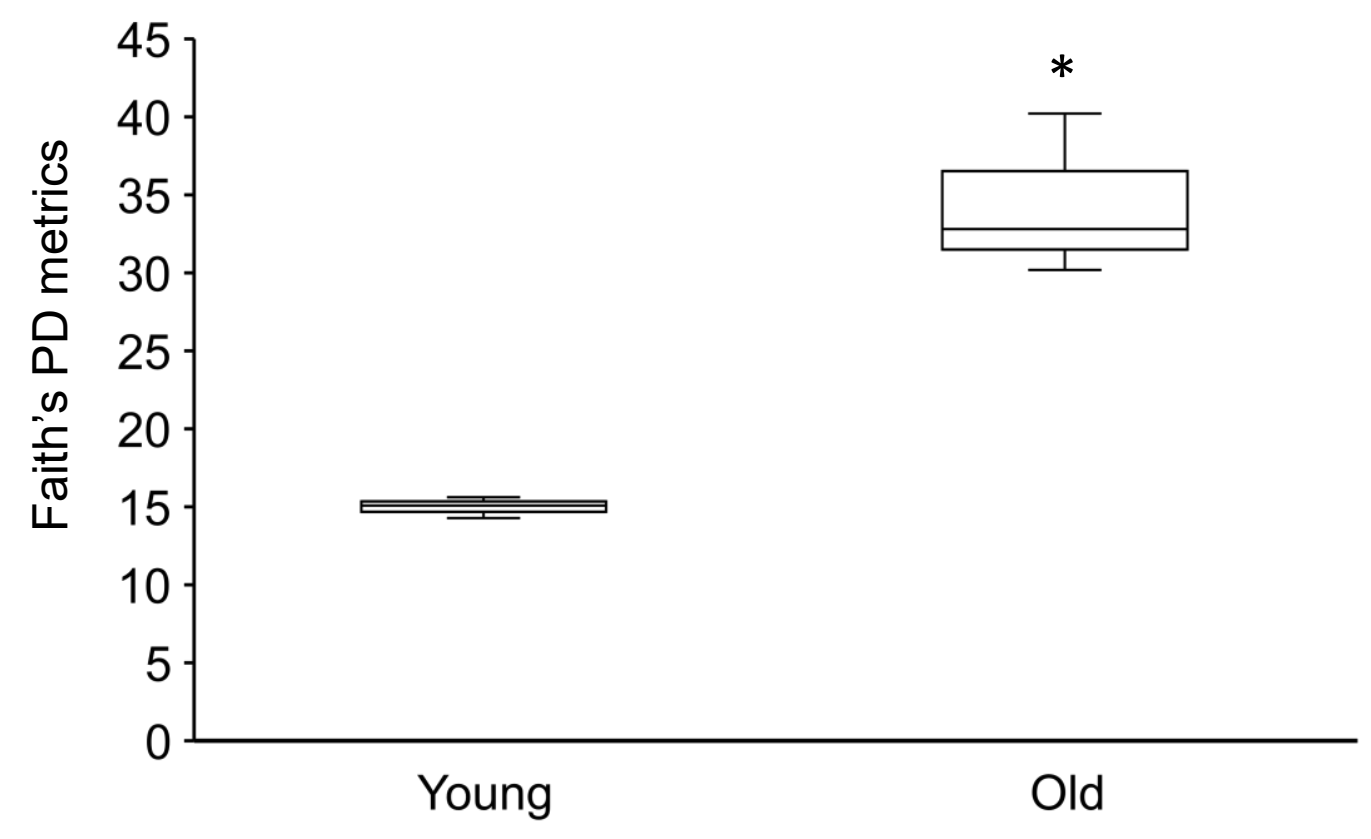

B

Axis $2(17.60 \%)$

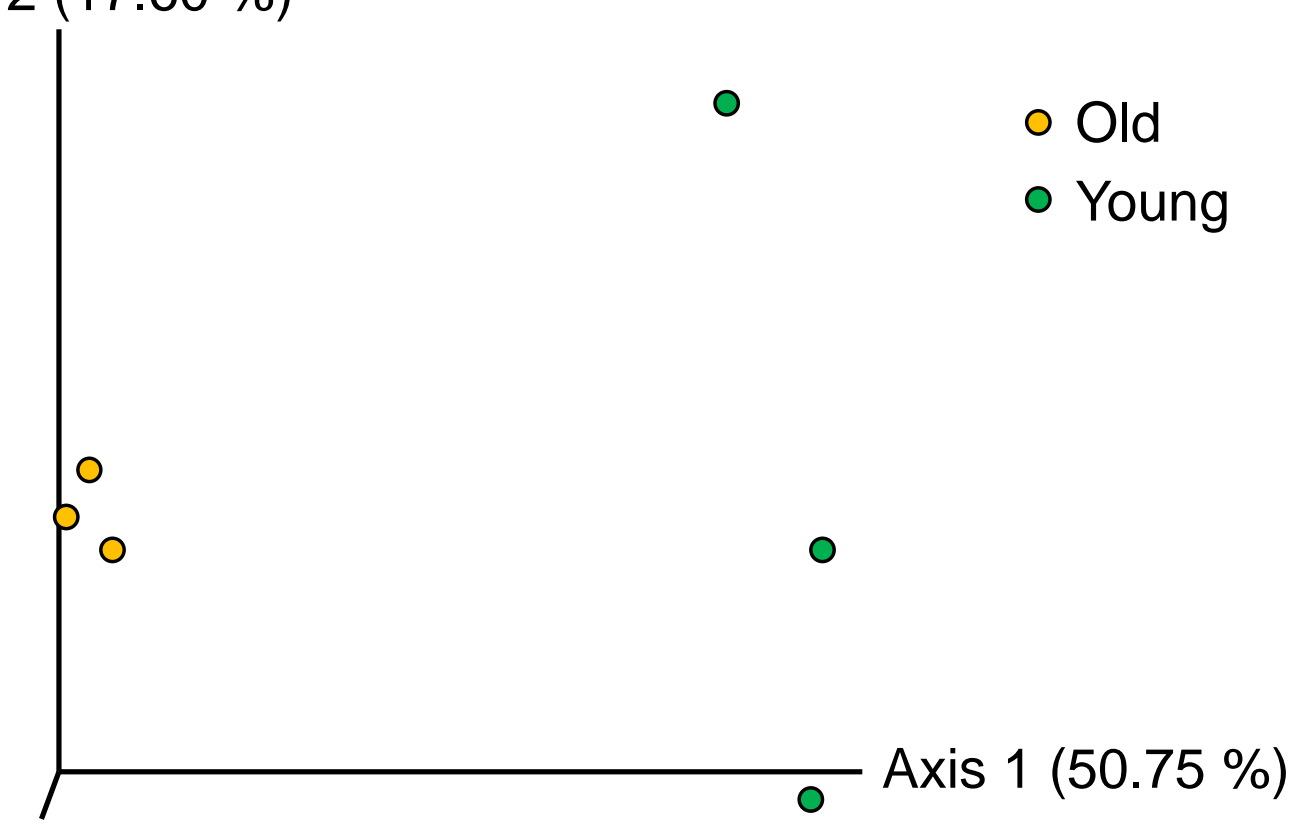

C

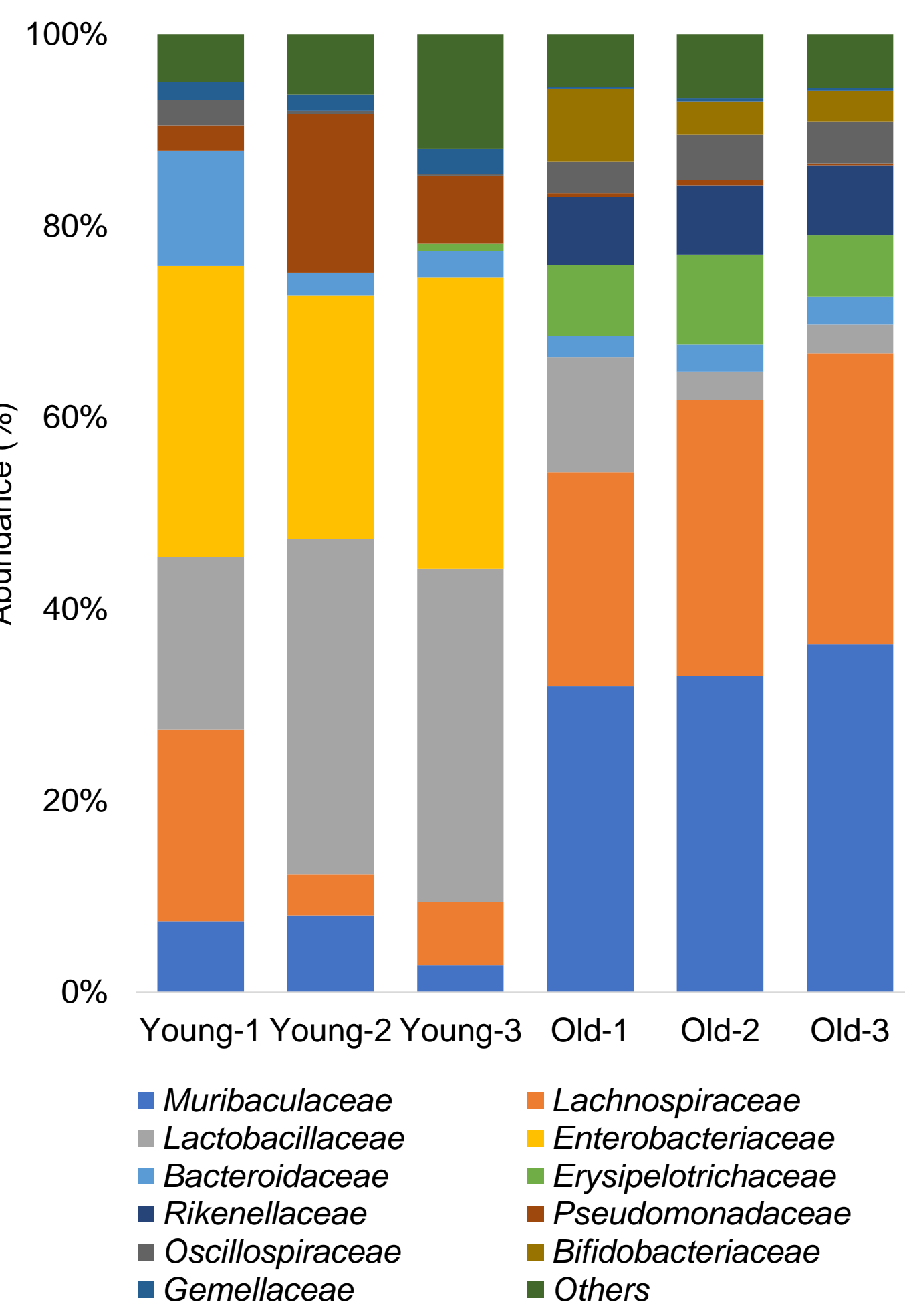

Axis $3(12.92 \%)$ 
Figure 5

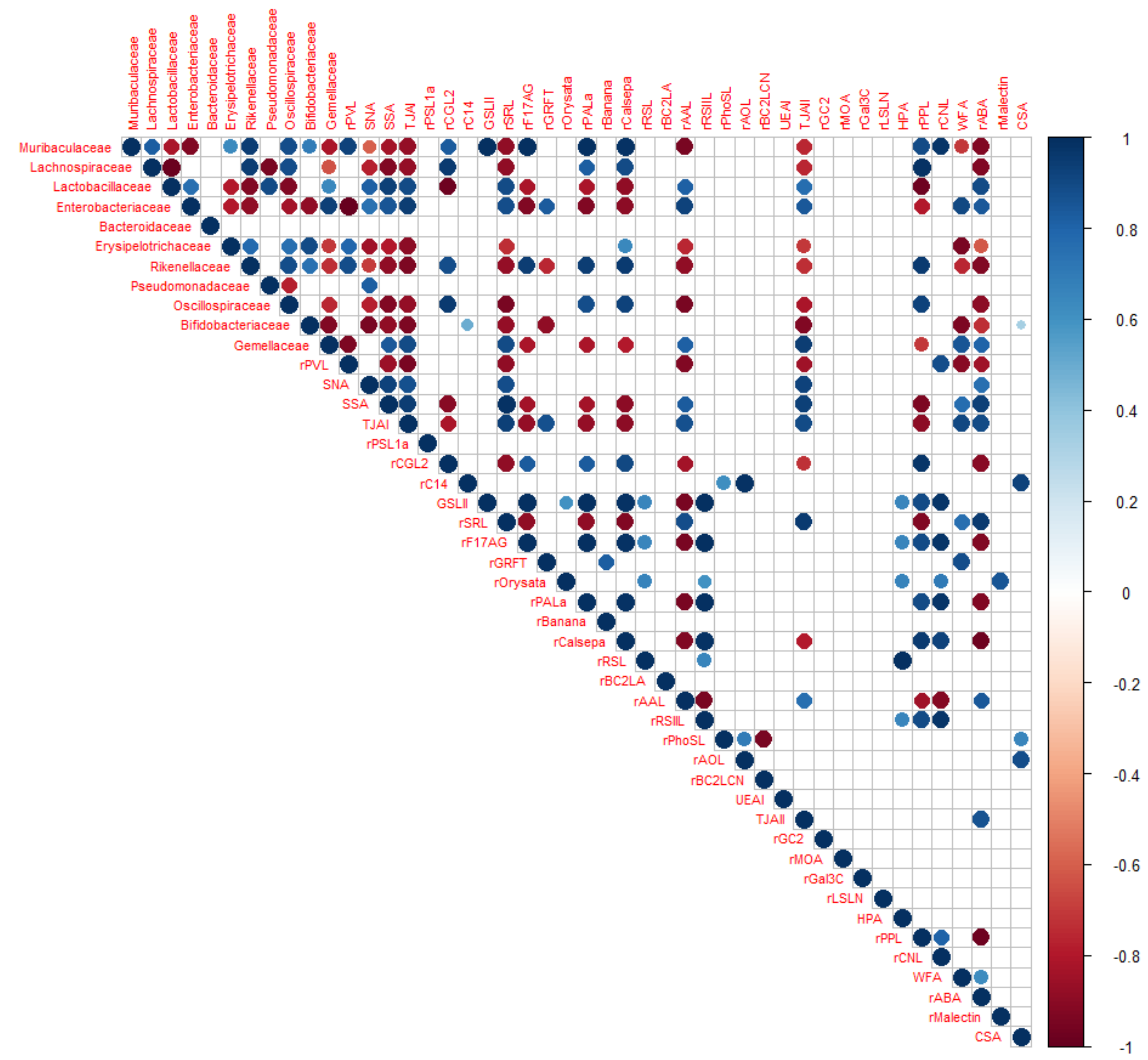




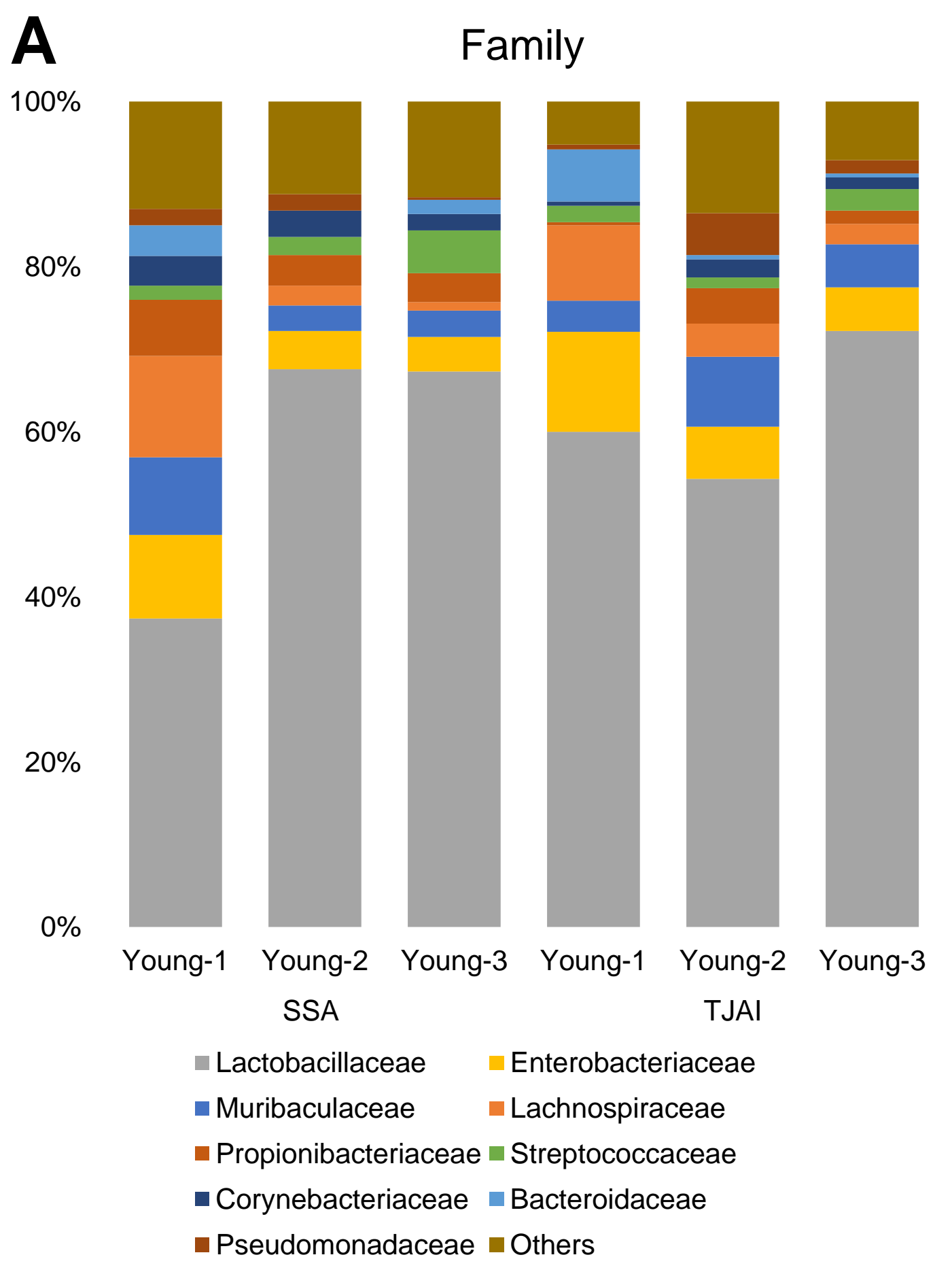

B

$100 \%$

$80 \%$

$60 \%$

$40 \%$

$20 \%$

$0 \%$
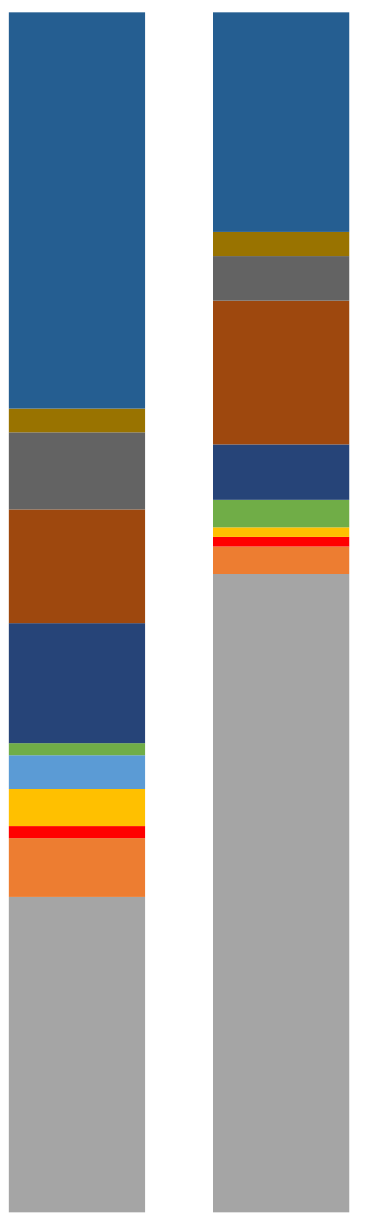

Young-1

Young-2 SSA

\section{Species}
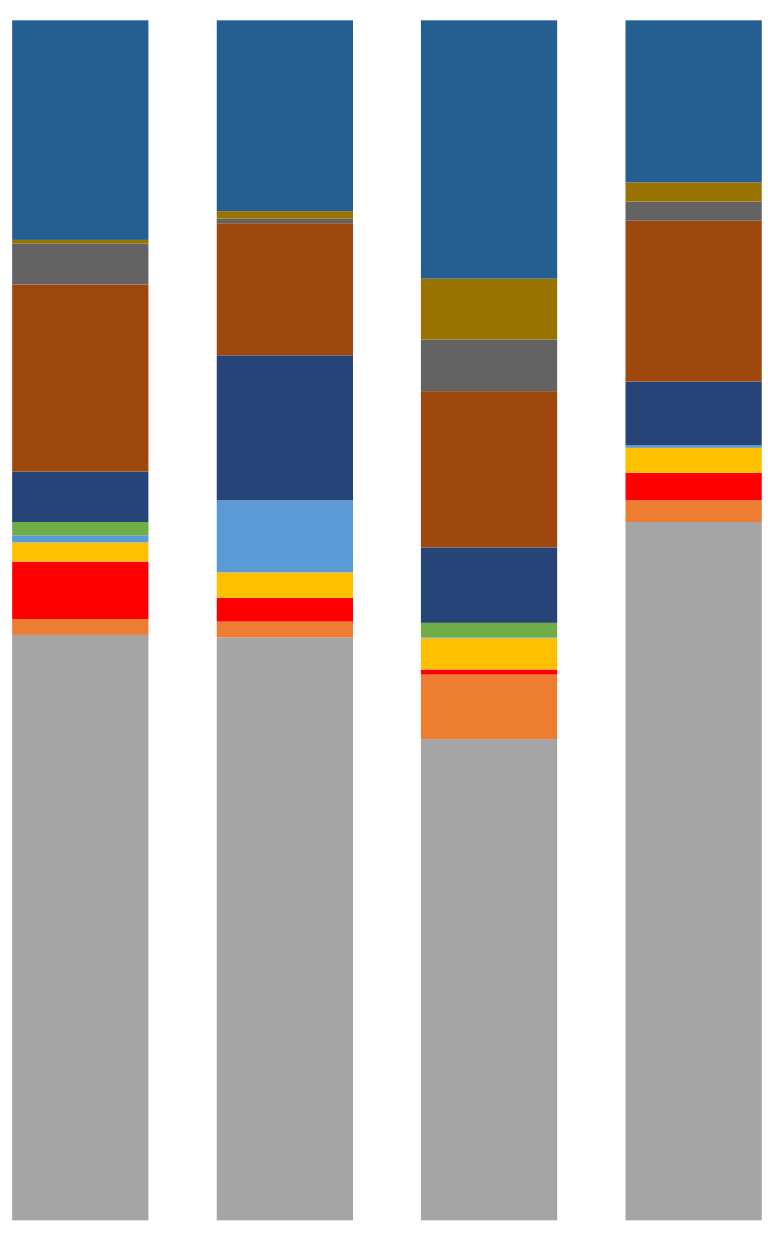

Young-3

Young-1

Young-2 TJAI

s_L_actobacillus_murinus $\square$ S_uncultured_Bacteroidales

-s_Streptococcus_danieliae $n$ s_uncultured_bacterium

s__Bacteroides_acidifaciens $\square$ ___Lactobacillus_johnsonii

-g_Escherichia-Shigella;__ g__Lactobacillus;

g_Cutibacterium;__ $\quad$ g__Pseudomonas;

- Others 